\title{
Direct contact membrane distillation with heat recovery: Thermodynamic insights from module scale modeling
}

\author{
Shihong Lin, Ngai Yin Yip, Menachem Elimelech* \\ Department of Chemical and Environmental Engineering, Yale University, P.O. Box 208286, New Haven, CT 06520-8286, United States
}

\section{A R T I C L E I N F O}

\section{Article history:}

Received 19 August 2013

Received in revised form

6 November 2013

Accepted 9 November 2013

Available online 18 November 2013

\section{Keywords:}

Membrane distillation

Direct contact membrane distillation

Modeling

Module

Energy efficiency

\begin{abstract}
A B S T R A C T
Direct contact membrane distillation (DCMD) can desalinate saline waters using low-grade heat and is thus economically attractive when low-temperature thermal energy is readily available. Coupling DCMD with a heat exchanger (HX) can significantly enhance the energy efficiency of the process by recovering the latent heat accumulated in the permeate (distillate) stream. This study evaluates the mass recovery rate (i.e., fraction of feed water recovered), $\gamma$, and the specific heat duty (i.e., energy input per unit mass of product water), $\beta$, of DCMD desalination using low-grade heat coupled with HX. Mass and heat transfer in DCMD and HX were modeled at the module scale and thermodynamic analysis of the system was performed. The relative flow rate (between the permeate and feed streams), $\alpha$, was found to be a critical operation parameter to optimize process performance, regardless of the mass and heat transfer kinetics. Both numerical evaluation and analytical analysis reveal a critical relative flow rate, $\alpha^{*}$, that demarcates DCMD operation between a permeate limiting regime (when $\alpha<\alpha^{*}$ ) and a feed limiting regime (when $\alpha>\alpha^{*}$ ), when mass transfer kinetics are not limiting. Similarly, we identified mass-limited and temperature-limited heat recovery regimes in the HX that are dependent on $\alpha$. Our analysis shows that the highest $\gamma$ and lowest $\beta$ achievable are solely determined by the thermodynamic properties of the system and always occur at the critical relative flow rate, $\alpha^{*}$. For example, the thermodynamic limits for $\gamma$ and $\beta$ are $6.4 \%$ and $27.6 \mathrm{~kJ} \mathrm{~kg}^{-1}$, respectively, for seawater desalination by single-pass DCMD at $60{ }^{\circ} \mathrm{C}$ with HX. However, in practical operation, as the DCMD membrane area and permeability cannot be infinitely large, the process is in a mass-transfer-limiting-regime and performance departs from the thermodynamic limits. Lastly, we demonstrate that heat transfer across a thermally-conductive DCMD membrane further reduces the recovery rate and energy efficiency of the process. The findings from this study have important implications for optimization of the DCMD process and for serving as criteria to assess process performance.
\end{abstract}

(c) 2013 Elsevier B.V. All rights reserved.

\section{Introduction}

Membrane distillation (MD) is a thermal separation process with a broad range of potential applications, including seawater desalination, treatment of high salinity brines, wastewater reuse, food processing, and biomedical applications [1,2]. More recently, MD has also been proposed as a key thermal separation component in hybrid membrane systems for wastewater treatment $[3,4]$, conversion of low-grade thermal energy to electricity [5], and protein purification [6]. General advantages of MD include high rejection of non-volatile components, low operation pressure

Abbreviations: MD, membrane distillation; DCMD, direct contact membrane distillation; HX, heat exchanger; PLR, permeate limiting regime (in MD); FLR, feed limiting regime (in MD); MTLR, mass transfer limiting regime (in MD); $\mathrm{PR}$, performance ratio, or mass of distillate (in $\mathrm{kg}$ ) produced per $2326 \mathrm{~kJ}$ heat input

* Corresponding author. Tel.: +1 203432 2789; fax: +1 2034324387.

E-mail address: menachem.elimelech@yale.edu (M. Elimelech). compared to reverse osmosis, low operation temperature and smaller foot print compared to conventional thermal separation processes [7]. The application of MD is particularly attractive when low-grade waste heat [8] or renewable thermal energy is readily available [9].

The operation of MD relies on the use of a hydrophobic membrane that serves both as the barrier for mass transfer of the liquid phase, and as the medium for mass transfer of the vapor phase $[1,10]$. In direct contact membrane distillation (DCMD), a microporous hydrophobic membrane is employed to separate a hot liquid stream (feed) and a cold liquid stream (permeate or distillate) [11,12]. The temperature difference between the feed and the permeate streams results in a difference in partial vapor pressure of water at the membrane-liquid interface, which is the driving force for the mass transfer across the membrane. In DCMD, there is no apparent phase transition because both the vaporization and the condensation occur microscopically within the porous membrane matrix. However, other MD configurations 
involve an observable, macroscopic condensation process, either inside the module as in air-gap MD (AGMD) [13], or in an external condenser as in sweeping-gas MD (SGMD) [14] and vacuum MD (VMD) [15]. DCMD is more suitable for desalination compared to SGMD or VMD if the solutes are non-volatile and the solvent (water) is the permeate [2]. Due to its relative simplicity for both experimental setup and industrial implementation, and its applicability for desalination, DCMD has been most intensively studied among all MD configurations [16].

In DCMD, mass and heat transfers occur simultaneously across the membrane. While the mass transfer is driven solely by the trans-membrane partial vapor pressure difference, there are two major pathways for heat transfer: convective heat transfer carried by the vapor flux and conductive heat transfer via the membrane/ vapor matrix [17]. Numerous studies have been conducted on modeling the DCMD process, with the majority devoted to understanding the coupled mass and heat transfer across the membrane and the membrane-liquid boundary layers [17-24]. Bench-scale studies with membrane coupons have also been carried out to corroborate the theoretical findings [18,21,25-29].

Comparatively, few studies have been reported on modeling DCMD at a module scale [30-34]. Module scale modeling is important, not only because in practice DCMD operates in modules, but also because it is necessary for estimating the energy efficiency for a DCMD system coupled with heat exchanger (HX) for heat recovery, which cannot be obtained from analysis of local mass and heat transfer alone. Implementation of HX to recover the latent heat of vaporization is critical for the DCMD process to become energy efficient [35].

In this paper, we carry out a module scale thermodynamic analysis of DCMD that fully incorporates the simultaneous heat and mass transfer processes. The analysis is first performed with only intrinsic heat transfer through a perfectly thermal-insulating MD membrane to identify the thermodynamic limits of the process. The efficacy of implementing an HX to recover the accumulated heat in the permeate stream effluent is also quantified by solving the mass and heat transfer equations in the coupled MD-HX system. Lastly, conductive heat transfer in an actual system is incorporated into the module analysis by employing thermally-conductive MD membranes, to examine the detrimental impact of heat conduction on the mass recovery rate and overall heat efficiency of the MD-HX system. Our analysis provides important insights into the thermodynamic limits of the DCMD process and the resulting impact on mass recovery and energy efficiency.

\section{Theoretical development}

\subsection{System overview of direct contact membrane distillation with heat exchange}

A schematic diagram of an MD-HX system for seawater desalination is presented in Fig. 1A. The influent feedwater $S_{0}$ (with mass flow rate $Q_{0}$ and temperature $T_{0}$ ) enters the HX as the cold stream. The MD permeate effluent $S_{4}$, which accumulates the latent heat of vaporization and is thus hot, enters the HX as the hot stream. Note that both the MD and HX modules operate in a counter-current flow mode to maximize the efficiency of mass and heat transfer, respectively. Heat is exchanged from the hot stream $S_{4}$, which is cooled to $S_{5}$ in the process, to the cold feedwater stream $S_{0}$, which warms up to $S_{1}$ in the $\mathrm{HX}$. The effluent temperature $T_{1}$ of the cold stream in HX may be still lower than the working temperature $T_{H}$ (i.e., temperature of the heat source) specified for the MD feed, in which case extra heat has to be supplied by an external heat source to bring $T_{1}$ to the influent temperature of the MD feed $\left(T_{2}=T_{H}\right)$. In a well-designed MD module, the majority of the heat in the feed will be transferred to the permeate stream by both convective and conductive heat transfer and the effluent of the MD feed will cool down considerably. For a desalination process, the concentrated effluent of the MD feed $\left(S_{3}\right)$ can either be released from the system as a waste stream, or it can be recycled as the influent.

The effluent stream of the MD permeate, which accumulates the heat transferred from the MD feed, enters the HX as the hot influent stream, $S_{4}$. The HX hot stream cools down from $T_{4}$ to $T_{5}$ by giving up heat to the cold feed stream. The amount of water transferred from the MD feed to the MD permeate as vapor is then taken as product water $\left(S_{7}\right)$ from the effluent of the HX hot stream $\left(S_{5}\right)$. The remaining portion of the HX hot stream effluent is then recirculated back to the MD module as the permeate influent $\left(S_{8}\right)$, which serves as both the coolant and the vapor collector in a DCMD process. In a well-designed MD-HX system, where mass and heat transfer are not limiting, the effluent temperature of the
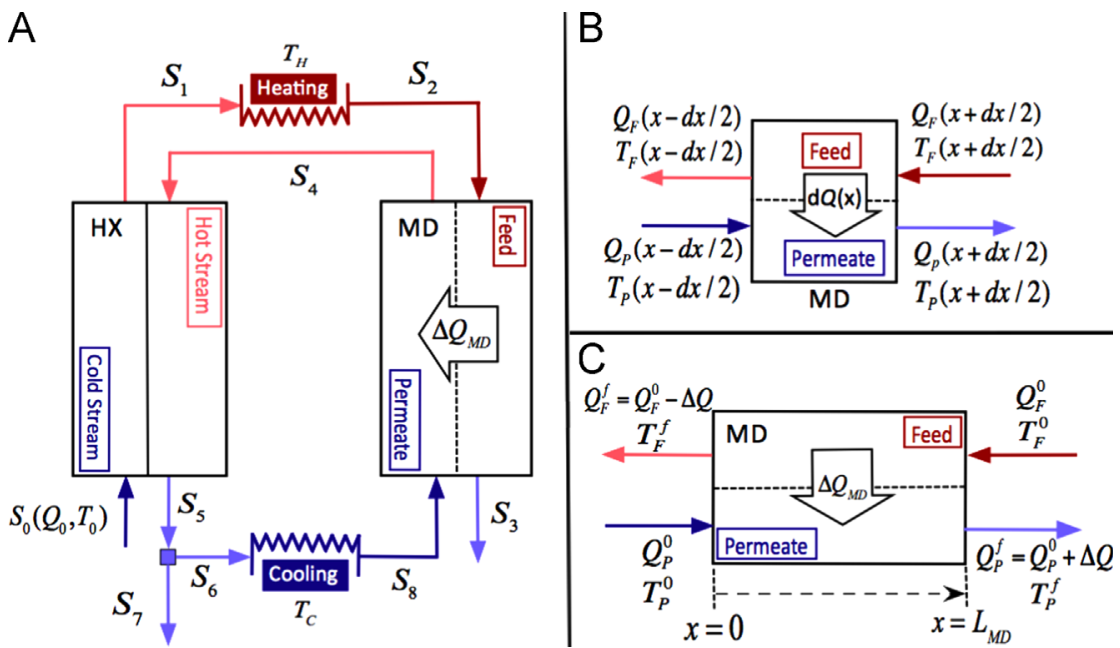

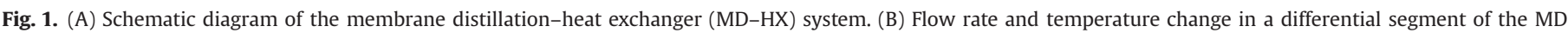

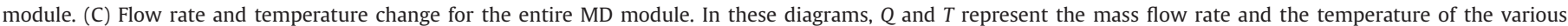

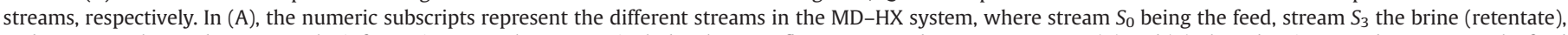

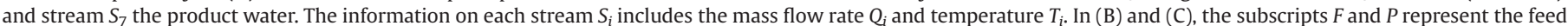
and permeate (distillate), respectively, and the superscripts $f$ and 0 represent the final and initial conditions, respectively. 
HX hot stream $\left(T_{5}=T_{6}\right)$ should be close to the temperature of the heat sink $\left(T_{C}\right)$. Otherwise, the surplus heat needs to be surrendered to the heat sink to further decrease the temperature from $T_{6}$ to that of the influent of MD permeate $\left(T_{8}=T_{C}\right)$ in order to maintain maximum possible driving force in the MD operation and steady state operation.

In this study, we simplify the heat source as a device that brings the temperature of a stream $\left(<T_{H}\right)$ to the specified heat source temperature, $T_{H}$, by providing the corresponding amount of heat $q_{H}$. Likewise, the heat sink is simplified as a device that brings the temperature of a stream ( $>T_{C}$ ) to the specified heat sink temperature, $T_{C}$, by extracting the corresponding amount of heat, $q_{C}$. The simplified heat source and heat sink can also be considered as a heat reservoir of constant temperature as often assumed in thermodynamic analyses. In actual operation, employing nonideal heat exchangers to transfer energy would necessitate a finite temperature difference between the streams to maintain an adequate driving force. Therefore, in practice, $T_{H}$ will be lower than the real temperature of the incoming heating stream, and $T_{C}$ will be higher than the real temperature of incoming cooling stream.

\subsection{Local mass transfer in the MD module}

For each differential segment of the MD module (Fig. 1B), the local mass balance can be described as

$\frac{d Q_{F}(x)}{d x}=\frac{d Q_{P}(x)}{d x}=\frac{A_{M D}}{L_{M D}} J_{m}(x)$

where $Q_{F}(x)$ and $Q_{P}(x)$ are the mass flow rates of the feed and permeate streams at axial position $x$ in the MD module, respectively, $A_{M D}$ is the total membrane area in the MD module, $L_{M D}$ is the length of the MD module, and $J_{m}(x)$ is the local transmembrane mass flux. The latter is determined by both the driving force and permeability of the membrane.

Although the mechanistic driving force for trans-membrane mass transfer is the partial vapor pressure difference $\Delta P_{W}$, it is more convenient to use the temperature difference $\Delta T$ as the proxy driving force for this analysis. Note that $\Delta P_{w}$ is not strictly linear with $\Delta T$ based on the Clausius-Clapeyron relation or on the Antoine equation; however, the linearization approximation is sufficient when $\Delta T$ is not too large [17], which is readily satisfied in most well-designed DCMD systems. We can thus relate the local mass flux $J_{m}(x)$ to the local bulk temperatures of the feed, $T_{F}(x)$, and the permeate, $T_{P}(x)$, using a phenomenological relation

$J_{m}(x) \approx K_{m, M D}\left[T_{F}(x)-T_{P}(x)-\Delta T_{t h}(x)\right]=K_{m, M D}\left[\Delta T(x)-\Delta T_{t h}(x)\right]$

where $K_{m, M D}$ is the empirical mass transfer coefficient in the DCMD process, $\Delta T(x)$ is the bulk temperature difference between the feed and the permeate at position $x$, and $\Delta T_{t h}(x)$ is the threshold temperature difference which is discussed later. It should be noted that $K_{m, M D}$ accounts for the permeability of the membrane as well as the effect of the flow hydrodynamics on mass transfer and is thus also a property of the operation conditions. Even though $K_{m, M D}$ is not an intrinsic property of the membrane and may vary along the membrane module, using an empirical mass transfer coefficient in our modeling is advantageous because (i) it can be directly calculated from experimental results (from the slope of $J_{m}$ as a function of $\Delta T$ ) and (ii) the effect of temperature polarization (TP) is already accounted for in the $K_{m, M D}$. On the other hand, the assessment of $\Delta P_{W}$ requires the knowledge of the degree of TP, which is more difficult to estimate accurately along the membrane channel and renders the theoretical analysis unnecessarily unwieldy.

The $\Delta T_{t h}(x)$ term in Eq. (2) is the threshold temperature difference, which accounts for the fact that the presence of solute in the feed solution lowers the partial vapor pressure of water at the feed-membrane interface [36]. The trans-membrane partial vapor pressure difference vanishes when $\Delta T$ reaches $\Delta T_{t h}(x)$ and mass transfer no longer occurs. Mathematically, $\Delta T_{t h}(x)$ is given by

$\Delta T_{t h}(x)=T_{F}^{*}(x)-T_{P}(x)$

where $T_{F}^{*}(x)$ is the threshold feed temperature defined such that

$p_{W}\left(C(x), T_{F}^{*}(x)\right)=p_{W}\left(0, T_{P}(x)\right)$

here $p_{W}$ is the partial vapor pressure of water as a function of temperature and solute concentration, and $C(x)$ is the solute concentration of the feed at position $x$. That is, the feed stream at temperature $T_{F}^{*}$ and concentration $C$ has the same partial vapor pressure as the permeate (distillate) stream $(C=0)$ at temperature $T_{p}$. The equality expressed in Eq. (4) represents the condition of zero driving force. Similarly, $\Delta T_{t h}(x)$ can also be defined as

$\Delta T_{t h}(x)=T_{F}(x)-T_{P}^{*}(x)$

where $T_{P}^{*}(x)$ is the threshold permeate temperature defined such that

$p_{W}\left(C(x), T_{F}(x)\right)=p_{W}\left(0, T_{P}^{*}(x)\right)$

We note that although the effect of TP can be accounted for by using an empirical $K_{m, M D}$ that relates $J_{m}(x)$ to $\Delta T, \Delta T_{t h}(x)$ is defined based on the bulk temperatures, which implicitly assumes the absence of TP and is thus valid only when TP is negligible. In other words, with TP in actual practice, $\Delta T_{t h}(x)$ should be larger than what is calculated using Eqs. (3) and (4) (or (5) and (6)) to an extent that depends on the actual degree of TP.

\subsection{Local heat transfer in the MD module}

\subsubsection{Heat transfer across the membrane}

Heat transfer in MD involves simultaneous convective and conductive transfer mechanisms. At any axial location along the MD module, the heat flux, $q_{m}$, which is constant across the membrane (direction $z$ ) at steady state, is given by [19]

$q_{m}=J_{m} h_{V}(T(z))-k_{c, M D} \frac{d T(z)}{d z}$

where $J_{m}$ is the mass flux of vapor as given by Eq. (2), $h_{V}$ is the specific enthalpy of vapor, which is a function of temperature, and $k_{c, M D}$ is the heat conductivity of the membrane-vapor matrix, which includes contributions from the vapor/air and membrane material.

The enthalpy of the vapor as a function of temperature can be expressed as

$h_{V}(T)=h_{V}\left(T_{0}\right)+\int_{T_{0}}^{T} c_{p, V} d T$

where $h_{V}\left(T_{0}\right)$ is the specific enthalpy of vapor at reference temperature $T_{0}$ and $c_{p, V}$ is the specific heat of the water vapor. Another way to interpret $h_{V}(T)$ that better reflects the process of evaporation is to view it as the sum of the specific enthalpy of vaporization of pure water $h_{v a p, w}(T)$ and the enthalpy of the liquid water $h_{L, W}(T)$ :

$h_{V}(T)=h_{v a p, W}(T)+h_{L, W}\left(T_{0}\right)+\int_{T_{0}}^{T} c_{p, L} d T$

where $h_{L, W}\left(T_{0}\right)$ is the enthalpy of liquid water at reference temperature $T_{0}$ and $c_{p, L}$ is the specific heat of the liquid water. The reference temperature $T_{0}$ is assumed to be $0{ }^{\circ} \mathrm{C}$, such that $h_{L, W}=0$ [37].

In the temperature range usually encountered in $\mathrm{MD}$, the heat capacities of the liquid, $c_{p, L}$, and the vapor, $c_{p, V}$, can be treated as constants, so that Eqs. (8) and (9) can be simplified to linear 
functions of the temperature:

$h_{V}(T) \approx h_{V}\left(T_{0}\right)+c_{p, V}\left(T-T_{0}\right)$

and

$h_{L, W}(T)=h_{L, W}\left(T_{0}\right)+c_{p, L}\left(T-T_{0}\right)$

By solving Eq. (7) using the linear form of $h_{V}(T)$ as given by Eq. (10), the constant heat flux can be written as a function of the mass flux and the temperatures of the feed and of the permeate

$q_{m}=J_{m}\left(A-\frac{T_{F} \exp \left(B J_{m}\right)-T_{P}}{\exp \left(B J_{m}\right)-1} c_{p, V}\right)$

where $A$ is constant defined as

$A=h_{V}\left(T_{0}\right)-c_{p, V} T_{0}$

and $B$ is a parameter given by

$B=\frac{c_{p, V} \delta}{k_{c, M D}}$

with $\delta$ being the thickness of the membrane.

Using Eqs. (7), (10), and (12), the conductive heat transfer at the permeate-membrane interface, $\zeta_{P}$, can then be written as

$\zeta_{P}=-\left.k_{c, M D} \frac{d T}{d z}\right|_{T=T_{P}}=q_{m}-J_{m} h_{V}\left(T_{P}\right)=\frac{J_{m} c_{p, V} \exp \left(B J_{m}\right)}{\exp \left(B J_{m}\right)-1}\left(T_{F}-T_{P}\right)$

Although not explicitly expressed here, $T_{F}, T_{P}$, and $J_{m}$ are local variables that depend on the axial position $x$ along the module. Similarly, the conductive heat transfer at the feed-membrane interface, $\zeta_{F}$, can be expressed as

$\zeta_{F}=-\left.k_{c, M D} \frac{d T}{d z}\right|_{T=T_{F}}=q_{m}-J_{m} h_{V}\left(T_{F}\right)=\frac{J_{m} c_{p, V}}{\exp \left(B J_{m}\right)-1}\left(T_{F}-T_{P}\right)$

Note that as long as the conductive heat transfer is in the same direction as the mass flux, $\exp \left(B J_{m}\right)$ is always greater than 1 . The difference between the conductive heat transfers at the permeatemembrane interface and the feed-membrane interface, $\Delta \zeta$, is given by

$\Delta \zeta\left(J_{m}, T_{F}, T_{P}\right)=-\left(\left.k_{c, M D} \frac{d T}{d z}\right|_{T=T_{P}}-\left.k_{c, M D} \frac{d T}{d z}\right|_{T=T_{F}}\right)=J_{m} c_{p, V}\left(T_{F}-T_{P}\right)$

which is the difference between the enthalpy of vapor at $T_{F}$ and that at $T_{P}$, according to Eqs. (7) and (10).

The implication of Eq. (17) is that conductive heat transfer is not only inevitable in practice (i.e., even with a perfectly insulating membrane material, the vapor itself is still conductive), but it is also a necessary condition for the mass transfer to occur. In the extreme case where the membrane is extremely insulating (e.g., $\delta \rightarrow \infty$ or $k_{c, M D} \rightarrow 0$ ), there will be almost no conductive heat transfer at the feed-membrane interface, but the conductive heat transfer at the permeate-membrane interface is then equal to $\Delta \zeta$ given by Eq. (17).

\subsubsection{Heat flow along the MD module}

To model the simultaneous heat and mass transfer along the MD module, we couple the heat flux across the membrane with the axial heat flows in the feed and permeate channels. In any differential segment, the heat flux arriving at the permeate stream is simply $q_{m}$ as described by Eq. (12). The arriving heat flux consists of three components: (i) enthalpy of condensation, which is exactly the same as the specific enthalpy of vaporization at the same temperature (i.e. $h_{\text {vap }, w}(T)$ ); (ii) the inherent enthalpy of the liquid water transferred to the permeate $\left(h_{L, W}(T)=h_{L, W}\left(T_{0}\right)+\right.$ $c_{p, L}\left(T-T_{0}\right)$ ); and (iii) the conductive heat transfer $\left(\zeta_{P}\right)$ as given by Eq. (15).
The net change in total enthalpy of the permeate in a differential segment of the membrane channel is therefore

$\frac{d\left[Q_{P}(x) h_{L}\left(0, T_{P}(x)\right)\right]}{d x}=\frac{d Q_{P}(x)}{d x}\left[h_{v a p}\left(0, T_{P}(x)\right)+h_{L}\left(0, T_{P}(x)\right)\right]+\frac{A_{M D}}{L_{M D}} \zeta_{P}(x)$

For universality, hereinafter we replace $h_{v a p, w}(T)$ with $h_{v a p}(0, T)$, where $h_{\text {vap }}(C, T)$ is the specific enthalpy of vaporization of an $\mathrm{NaCl}$ solution of concentration $C$ (in molality, $\mathrm{mol} \mathrm{kg}^{-1}$ ) at temperature $T$ ( $C=0$ for pure water in permeate). We also rewrite the specific enthalpy of pure liquid water in the form of the specific enthalpy of the $\mathrm{NaCl}$ solution $h_{L}(C, T)$, with the concentration of $\mathrm{NaCl}$ being 0 . The $Q_{P}(x)$ is included in the differential in Eq. (18) because the permeate flow rate changes along the module due to mass transfer.

Similar to Eq. (18), we can write an equation for the local energy balance accounting for the three heat transfer contributions at the feed-membrane interface:

$\frac{d\left[Q_{F}(x) h_{L}\left(C(x), T_{F}(x)\right)\right]}{d x}=\frac{d Q_{F}(x)}{d x}\left[h_{\text {vap }}\left(C(x), T_{F}(x)\right)+h_{L}\left(0, T_{F}(x)\right)\right]+\frac{A_{M D}}{L_{M D}} \zeta_{F}(x)$

where $h_{L}\left(C(x), T_{F}(x)\right)$ is the specific enthalpy of the feed $\mathrm{NaCl}$ solution at concentration $C(x)$ and temperature $T_{F}(x)$, and $h_{L}(0$, $T_{F}(x)$ ) is the specific enthalpy of pure liquid water at feed temperature $T_{F}(x)$. Note that the second term on the right-handside is $h_{L}\left(0, T_{F}(x)\right)$ instead of $h_{L}\left(C(x), T_{F}(x)\right)$ as on the left-hand-side, because vapor leaving the feed solution contains no salt; that is, if the vapor is condensed at the same temperature $T_{F}(x)$, the inherent specific enthalpy carried by the condensed liquid is $h_{L}\left(0, T_{F}(x)\right)$. We also note that the heat flux leaving the feed is slightly larger than $q_{m}$ as the enthalpy of vaporization is $h_{v a p}\left(C(x), T_{F}(x)\right)$ instead of $h_{\text {vap }}\left(0, T_{F}(x)\right)$, due to the presence of solute in the feed.

\subsection{Coupling heat transfer and mass transfer in the MD module}

The distributions of mass flow rates, $Q_{F}(x)$ and $Q_{P}(x)$, and of temperatures, $T_{F}(x)$ and $T_{P}(x)$, can be obtained by solving a system of four differential equations given by Eq. (1) (comprising both the feed and permeate sides), which represents the mass transfer, and Eqs. (18) and (19), which represent the heat transfer. All other thermodynamic properties involved in the equations (e.g., $h_{v a p}, h_{L}$, and $c_{p, L}$ ) are functions of temperature $T(x)$ and/or $\mathrm{NaCl}$ concentration $C(x)$, which, in turn, are dependent on the local flow rates based on mass balance.

With the phenomenological equation relating the transmembrane mass flux and the temperature difference (Eq. (2)), we can rewrite the system of the governing differential equations:

$$
\begin{aligned}
& \frac{d Q_{F}(x)}{d x}=\frac{A_{M D}}{L_{M D}} K_{m, M D}\left[T_{F}(x)-T_{P}(x)-\Delta T_{t h}(x)\right] \\
& \frac{d Q_{P}(x)}{d x}=\frac{A_{M D}}{L_{M D}} K_{m, M D}\left[T_{F}(x)-T_{P}(x)-\Delta T_{t h}(x)\right] \\
& \frac{d\left[Q_{F}(x) h_{L}\left(C(x), T_{F}(x)\right)\right]}{d x}=\frac{d Q_{F}(x)}{d x}\left[h_{\text {vap }}\left(C(x), T_{F}(x)\right)+h_{L}\left(0, T_{F}(x)\right)\right]+\frac{A_{M D}}{L_{M D}} \zeta_{F}(x)
\end{aligned}
$$

$\frac{d\left[Q_{P}(x) h_{L}\left(0, T_{P}(x)\right)\right]}{d x}=\frac{d Q_{P}(x)}{d x}\left[h_{v a p}\left(0, T_{P}(x)\right)+h_{L}\left(0, T_{P}(x)\right)\right]+\frac{A_{M D}}{L_{M D}} \zeta_{P}(x)$

We note that Eqs. (20-C) and (20-D) are the same as Eqs. (19) and (18), respectively. The repetition with new equation numbers is to 
facilitate the construction of a coherent system of equations (Eqs. (20-A)-(20-D)) that includes all the governing equations for mass and heat transfer.

In the ideal scenario where the membrane is perfectly thermalinsulating (i.e., $k_{c, M D} \rightarrow 0$ ), $\exp \left(B J_{m}\right) \rightarrow \infty$, and $\zeta_{F}(x)$ approaches 0 while $\zeta_{P}(x)$ approaches $J_{m} c_{p, v}\left(T_{F}-T_{P}\right)$. For this ideal case, the system of governing differential equations simplifies to

$\frac{d Q_{F}(x)}{d x}=\frac{A_{M D}}{L_{M D}} K_{m, M D}\left[T_{F}(x)-T_{P}(x)-\Delta T_{t h}(x)\right]$

$\frac{d Q_{P}(x)}{d x}=\frac{A_{M D}}{L_{M D}} K_{m, M D}\left[T_{F}(x)-T_{P}(x)-\Delta T_{t h}(x)\right]$

$\frac{d\left[Q_{F}(x) h_{L}\left(C(x), T_{F}(x)\right)\right]}{d x}=\frac{d Q_{F}(x)}{d x}\left[h_{\text {vap }}\left(C(x), T_{F}(x)\right)+h_{L}\left(0, T_{F}(x)\right)\right]$

$\frac{d\left[Q_{P}(x) h_{L}\left(0, T_{P}(x)\right)\right]}{d x}=\frac{d Q_{P}(x)}{d x}\left[h_{v a p}\left(0, T_{P}(x)\right)+h_{L}\left(0, T_{P}(x)\right)\right.$

$$
\left.+c_{p, V}\left(T_{F}(x)-T_{P}(x)\right)\right]
$$

For both cases, the boundary conditions (illustrated in Fig. 1C) are given by

$Q_{F}\left(L_{M D}\right)=Q_{F}^{0}$

$Q_{P}(0)=Q_{P}^{0}$

$T_{F}\left(L_{M D}\right)=T_{F}^{0}=T_{H}$

$T_{P}(0)=T_{P}^{0}=T_{C}$

\subsection{Heat transfer in the $H X$ module}

The heat exchange between the hot stream $\left(S_{4} \rightarrow S_{5}\right)$ and the cold stream $\left(S_{0} \rightarrow S_{1}\right)$ in the HX is a standard and well-understood heat transfer problem. The differential equations for the heat transfer are

$Q_{h} \frac{d h_{L}\left(0, T_{h}(x)\right)}{d x}=Q_{h} c_{p, L}(0) \frac{d T_{h}(x)}{d x}=K_{c, H X} \frac{A_{H X}}{L_{H X}}\left[T_{h}(x)-T_{c}(x)\right] \quad(23-\mathrm{A})$

$Q_{c} \frac{d h_{L}\left(C_{0}, T_{c}(x)\right)}{d x}=Q_{c} c_{p, L}\left(C_{0}\right) \frac{d T_{c}(x)}{d x}=K_{c, H X} \frac{A_{H X}}{L_{H X}}\left[T_{h}(x)-T_{c}(x)\right]$

where the hot stream flow rate, $Q_{h}$, is equal to the effluent flow rate of the MD permeate, $Q_{p}^{f}\left(Q_{4}\right.$ in Fig. $\left.1 \mathrm{~A}\right)$, and the cold stream flow rate, $Q_{c}$, is equal to the flow rate of the influent (feed stream) of the overall system $\left(Q_{0}\right.$ in Fig. $\left.1 \mathrm{~A}\right)$. Note that we use lower case " $h$ " and " $c$ " in the subscripts for $Q$ and $T$ to represent the hot and cold streams in HX, respectively, whereas capital " $H$ " and " $C$ " are used as subscripts to denote heat source and heat sink of the MDHX system.

Both flow rates are constant throughout the HX module due to the absence of mass transfer. The heat capacities of pure water (hot stream) and a $\mathrm{NaCl}$ solution at concentration $C_{0}$ (cold stream) are $c_{p, L}(0)$ and $c_{p, L}\left(C_{0}\right)$, respectively, and can be approximated as constants as they are weak functions of temperature. The heat transfer coefficient of the $\mathrm{HX}$ (plate or tube) is $K_{c, H X}$, the contact area of the HX is $A_{H X}$, and the length of the HX module is $L_{H X}$. The boundary conditions for Eqs. (23-A) and (23-B) are

$T_{h}\left(L_{H X}\right)=T_{P}^{f}$

$T_{C}(0)=T_{C}$
Boundary condition (24-A) indicates that the influent temperature of the hot stream in the HX is the effluent temperature (or "final" temperature) of the permeate stream in the MD module.

\subsection{Parameters quantifying process performance and energy efficiency}

Solving the governing differential equations for the mass and heat transfer in the MD module yields the flow rate and temperature distributions along the permeate and feed channels of the MD module. Similarly, solving the governing heat transfer differential equations for the HX yields the temperature distribution profiles of the hot and cold streams in the HX. To quantify the overall performance of the MD-HX system, we define the following performance parameters that can be calculated from the solved temperature and flow rate distributions.

The first performance parameter is the feed mass recovery rate (or simply "mass recovery rate"), $\gamma$, which is the fraction of feed (mass) flow rate that permeates through the membrane and adds to the permeate (mass) flow rate

$\gamma \equiv \frac{\Delta Q_{M D}}{Q_{F}^{0}}=\frac{A_{M D}}{L_{M D}} \frac{\int_{0}^{L_{M D}} J_{m}(x) d x}{Q_{F}^{0}}$

Note that $\gamma$ is characteristic of the MD module performance, but not of the overall MD-HX process. Therefore it is still a relevant DCMD performance indicator even if no heat recovery measure is implemented.

The second performance parameter is the specific heat duty, $\beta$, which is the amount of external heat energy $\left(q_{H}\right)$ supplied to the MD-HX system via the heat source to produce a unit mass of distilled water (as an increment in the permeate stream):

$\beta \equiv \frac{q_{H}}{\Delta Q_{M D}}=\frac{c_{p, L}\left(C_{0}\right) Q_{F}^{0}\left(T_{2}-T_{1}\right)}{\Delta Q_{M D}}$

This performance parameter $\beta$ can be expressed in the common units of $\mathrm{kJ} \mathrm{kg}^{-1}$. The more commonly used term in industry to quantify the energy efficiency of distillation is the performance ratio (PR), which is defined as the mass of distillate (in $\mathrm{kg}$ ) produced per $2326 \mathrm{~kJ}$ heat input [38]. PR is the metric version of the gain output ratio (GOR), another commonly used energy efficiency indicator for thermal desalination processes. The conversion between $\beta$ and PR is straightforward:

$\beta=\frac{2326}{P R}$

Specifically, in the system depicted in Fig. 1A, the heat energy supplied to the system is used to raise the effluent temperature of the cold stream in the HX $\left(T_{1}\right)$ to the specified working temperature $\left(T_{H}\right)$ or the influent temperature of the feed stream in the MD $\left(T_{2}\right)$. Combining Eqs. (25) and (26) yields the relation between $\beta$ and $\gamma$ :

$\beta=\frac{c_{p, L}\left(C_{0}\right)\left(T_{2}-T_{1}\right)}{\gamma}$

To achieve higher overall energy efficiency for the MD-HX process, $\beta$ needs to be minimized by maximizing the mass recovery rate in the MD module and, at the same time, maximizing the effluent temperature of the cold stream, $T_{1}$, so that minimal heat is required to bring the MD feed stream to the working temperature, $T_{H}$.

Alternatively, we can also define a performance parameter called the heat recovery efficiency $(\lambda)$ as the fraction of total heat required to elevate the temperature of the MD feed from $T_{C}$ (or $T_{0}$ ) to $T_{H}$ (or $T_{2}$ ) that is contributed by the recovered heat in the HX 
(Fig. 1A):

$\lambda \equiv 1-\frac{q_{H}}{c_{p, L}\left(C_{0}\right) Q_{F}^{0}\left(T_{2}-T_{0}\right)}=\frac{T_{1}-T_{0}}{T_{2}-T_{0}}$

Combining Eqs. (28) and (29) yields the relation between $\lambda, \beta$, and $\gamma:$

$\lambda=\frac{c_{p, L}\left(C_{0}\right)\left(T_{H}-T_{C}\right)-\beta \gamma}{c_{p, L}\left(C_{0}\right)\left(T_{H}-T_{C}\right)}$

After defining these important performance indicators $(\lambda, \beta$, and $\gamma$ ), we define an important operational parameter-the ratio of the permeate stream flow rate $\left(Q_{8}\right.$ in Fig. $\left.1 \mathrm{~A}\right)$ to the feed stream flow rate $\left(Q_{2}\right.$ in Fig. $\left.1 A\right)$, which we term the relative permeate flow rate (or simply "relative flow rate"), $\alpha$, of the MD module:

$\alpha \equiv \frac{Q_{P}^{0}}{Q_{F}^{0}}$

Being an operational parameter that is readily controlled, $\alpha$ is found to play a very significant role in determining the system performance, as will be shown in later discussions.

\section{Numerical solution of governing equations for the MD and HX processes}

The systems of differential equations governing the mass and heat transfer in the MD module (Eqs. (20) or (21)) were discretized using a standard finite volume method [39]. Specifically, the MD module was discretized into $n=100$ differential segments along the axial direction, which resulted in a system of $4 n$ nonlinear equations with $n$ sets of variables (each set includes $T_{F}(x), T_{P}(x)$, $Q_{F}(x)$, and $Q_{P}(x)$ ). This system of nonlinear equations was solved using the $f$ solve function in Matlab ${ }^{\circledR}$ with the default Trust-Region methods [40]. The system of differential equations governing the heat transfer in the HX module (Eq. (23)) is linear and thus was discretized to a system of linear equations that can be readily solved using the standard matrix method. The HX was discretized to $m=1000$, as a system of linear equations is significantly less computationally demanding to solve compared to a system of nonlinear equations. Accuracy of the results was not enhanced significantly with further refinement in the number of differential segments in either the MD or HX module.

The thermodynamic properties required to conduct the numerical modeling or analytical analysis are available in the literature in the form of correlation equations. The most important of these include the enthalpy of vaporization, $h_{\text {vap }}(C, T)$ [41,42], the enthalpy of $\mathrm{NaCl}$ solution, $h_{L}(C, T)$ [37], and the heat capacity of $\mathrm{NaCl}$ solution, $C_{p, L}(C, T)[43]$. The correlation equations and representative curves of these properties as a function of temperature and $\mathrm{NaCl}$ concentration are given in Appendix A.

The calculation of $\Delta T_{t h}(x)$ for a given concentration, $C$, and permeate temperature, $T_{P}$, requires an iterative process, which renders solving the system of non-linear equations computationally demanding. Therefore, $\Delta T_{t h}(x)$ as a function of $C$ and $T_{P}$ was first solved to yield the numerical results, which were then used to establish a correlation equation for $\Delta T_{t h}\left(C, T_{P}\right)$ (Appendix B). Throughout the study, we use a mass transfer coefficient $K_{m, M D}$ of $0.03 \mathrm{~kg} \mathrm{~m}^{-2} \mathrm{~min}^{-1}{ }^{\circ} \mathrm{C}^{-1}$ for the MD module, which is obtained from linear regression of the slope of $J_{m}$ as a function of $\Delta T$ of literature data of a typical commercially available DCMD membrane [29]. For the heat transfer coefficient in the HX, a typical value of $10^{3} \mathrm{~W} \mathrm{~m}^{-2}{ }^{\circ} \mathrm{C}^{-1}$ is chosen $[44,45]$.

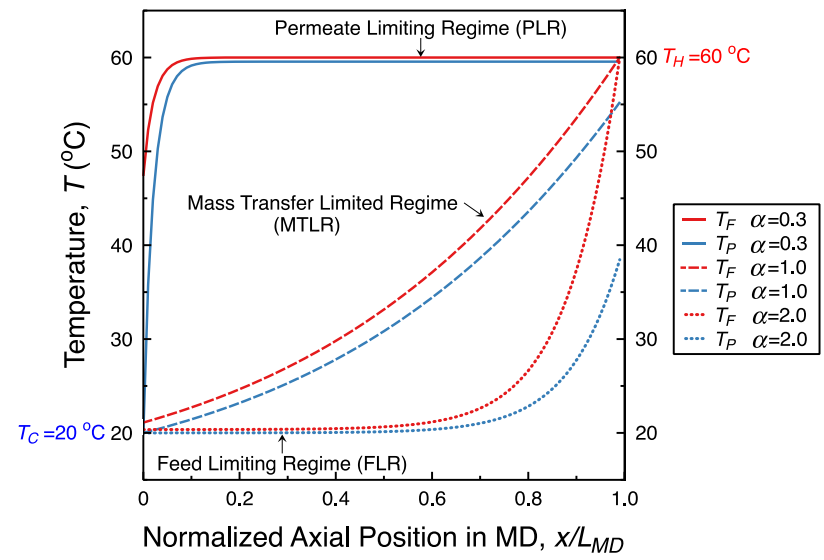

Fig. 2. Temperature distribution profiles for the three different operation regimes in an MD module. In the PLR, the permeate temperature reaches $T_{H}^{*}$ within the module. In the FLR, the feed temperature reaches $T_{C}^{*}$, within the module. In the transition regime (MTLR), neither the feed temperature reaches the $T_{C}^{*}$ nor the permeate temperature reaches $T_{H}^{*}$. Parameters used for calculations: $L_{M D}=2 \mathrm{~m}$, $\mathrm{A}_{M D}=50 \mathrm{~m}^{2}, K_{m, M D}=0.03 \mathrm{~kg} \mathrm{~m}^{2} \mathrm{~K}^{-1} \mathrm{~min}^{-1}{ }^{\circ} \mathrm{C}^{-1}$, and $Q_{F}^{0}=50 \mathrm{~kg} \mathrm{~min}^{-1}$. Note that $x / L_{M D}=0$ is at the entrance point of the permeate (distillate) stream, while $x / L_{M D}=1$ is the entrance point of the counter flow feed stream as depicted in Fig. 1C. (For interpretation of the references to color in this figure, the reader is referred to the web version of this article.)

\section{Results and discussion}

\subsection{Operation regimes in the MD module}

The MD-HX system was analyzed over a range of relative flow rates, $\alpha$, with a $0.6 \mathrm{~mol} \mathrm{~kg}^{-1} \mathrm{NaCl}$ feed solution at a temperature of $20{ }^{\circ} \mathrm{C}$ to represent seawater. The MD working temperature (i.e., the specified temperature of the influent feed stream) was set at $60{ }^{\circ} \mathrm{C}$. From the temperature distribution profiles along the axial direction of the MD module obtained from solving the governing equations for mass and heat transfer, we identified three distinct operation regimes, as described in the following discussions.

In the first scenario, which occurs when the relative flow rate $\alpha$ is low (i.e., low permeate flow rate compared to feed flow), the permeate (distillate) stream quickly warms up to $T_{H}^{*}$ due to its relatively lower flow rate (blue solid curve in Fig. 2), with $T_{H}^{*}$ being the temperature where

$p_{W}\left(C_{0}, T_{H}\right)=p_{W}\left(0, T_{H}^{*}\right)$

Note that $T_{H}$ is the temperature of the heat source and is thus equal to the influent temperature of the MD feed, $T_{F}^{0}$ (or stream $S_{2}$ in Fig. 1A).

At the point along the MD module where the permeate temperature $T_{P}$ reaches $T_{H}^{*}$, the trans-membrane temperature difference becomes equal to the threshold temperature $\Delta T_{\text {th }}$ (Eq. (5)). Beyond this point (in the direction of the permeate flow), the partial vapor pressure gradient vanishes and no mass transfer occurs along the remaining module length. Consequentially, the effluent temperature of the permeate $T_{P}^{f}$ is $T_{H}^{*}$. We define the operation of DCMD with such a temperature distribution profile as the permeate limiting regime (PLR) operation. The temperature profiles of the permeate and feed streams along the MD module for this operation regime are shown in Fig. 2 (solid blue and red lines).

In the opposite scenario, which occurs when $\alpha$ is high (i.e., high permeate flow rate relative to feed flow rate), the feed stream rapidly cools down and the feed temperature $T_{F}$ rapidly attains $T_{C}^{*}$ in the module (red dotted curves in Fig. 2), with $T_{C}^{*}$ being the temperature where

$p_{W}\left(C_{0}, T_{C}^{*},\right)=p_{W}\left(0, T_{C}\right)$ 
Note that $T_{C}$ is the temperature of the heat sink and is thus equal to the influent temperature of the MD permeate, $T_{P}^{0}$ (or stream $S_{8}$ in Fig. 1A). In this scenario, there is no trans-membrane flux beyond this point along the flow direction of the feed stream in the module as there is no driving force for mass transfer because $\Delta T$ is equal to $\Delta T_{t h}$ (Eq. (3)). We define the operation characterized by such a temperature distribution profile as the feed limiting regime (FLR) operation. The temperature profiles of the permeate and feed streams along the MD module for this operation regime are shown in Fig. 2 (dotted red and blue lines).

As the thermodynamic driving force for mass transfer vanishes within the module, both PLR and FLR are regarded as thermodynamically limited operation regimes. A somewhat analogous idea to these thermodynamic limiting regimes was proposed by Song et al. [46,47] for reverse osmosis desalination in a module. As the feed brine becomes concentrated along the reverse osmosis module, its osmotic pressure correspondingly increases to a point where it equals the applied pressure. Beyond this point along the reverse osmosis module, the driving force vanishes and the membrane area is unutilized. In the case of $\mathrm{MD}$, the depleted driving force in FLR or PLR is the difference in partial vapor pressure, and the operation can be limited by the flow rate of either the feed or permeate streams.

There is one more possible scenario in which $T_{F}$ and $T_{P}$ never reach $T_{C}^{*}$ and $T_{H}^{*}$, respectively, within the module (dashed blue and red curves in Fig. 2). In this case, the temperature difference at all points along the MD module is always greater than the threshold temperature difference as defined by either Eq. (3) or (5), and vapor flux occurs throughout the entire membrane length. We define this operation as the mass transfer limiting regime (MTLR). As we discuss later in the paper, the MTLR emerges only when the mass transfer is limited (i.e., membrane area is insufficient or the mass transfer coefficient is too low). In other words, if mass transfer is not a limiting factor, the MD operation is always in the thermodynamic limiting regimes of either PLR or FLR.

We note that a given $\alpha$ does not automatically classify the operation as PLR or FLR. MD operation can still be considered in MTLR with insufficient membrane area, even when $\alpha$ is very small or very large. As an example, an MD operation on a lab-scale small membrane coupon with a reasonable flow rate is always in MTLR, regardless of $\alpha$. However, less membrane area is required for the operation to be in PLR if $\alpha$ is very small, or to be in FLR if $\alpha$ is very large.

\subsection{Thermodynamic analysis of the MD operation regimes}

Numerical solution of the governing mass and heat transfer equations yields the temperature and flow rate distributions along the module, which allow us to identify the operation regime and calculate the mass recovery rate, $\gamma$. The conditions for the occurrence of the three operational regimes, PLR, FLR, and MTLR, can be more readily examined through an analytical examination of the DCMD process. The underlying conditions for the derivation are the following: when the operation is in PLR, $T_{P}^{f}=T_{H}^{*}$ and $T_{F}^{f} \geq T_{C}^{*}$, and analogously, when the operation is in FLR, $T_{F}^{f}=T_{C}^{*}$ and $T_{P}^{f} \leq T_{H}^{*}$. The key results of our analytical analysis are summarized in the following sections, with the detailed derivations presented in Appendix C.

\subsubsection{Permeate limiting regime (PLR)}

Our analytical examination (Appendix C) indicates that for MD operation in the PLR, the relative flow rate parameter, $\alpha$, has to be lower than the critical relative flow rate, $\alpha_{P L R}$ :

$\alpha \leq \alpha_{P L R}=\frac{T_{H}-T_{C}^{*}}{T_{H}^{*}-T_{C}} \frac{\left(\bar{h}_{v a p, P} / c_{p, P}\right)-\left(\left(T_{H}^{*}-T_{C}\right) / 2\right)}{\left(\bar{h}_{v a p, P} / c_{p, F}\right)+\left(c_{p, P} / c_{p, F}\right)\left(\left(T_{H}+T_{c}^{*}\right) / 2\right)-T_{C}^{*}}$ where $c_{p, P}$ is the heat capacity of the permeate (which is equal to $\left.c_{p, L}(0)\right), c_{p, F}$ is the heat capacity of the feed (which is approximately equal to $\left.c_{p, L}\left(C_{0}\right)\right)$, and $\bar{h}_{v a p, P}$ is the average enthalpy of vaporization of the permeate and can be approximated by

$\bar{h}_{\text {vap }, P} \approx \frac{h_{\text {vap }}\left(0, T_{H}^{*}\right)+h_{\text {vap }}\left(0, T_{C}\right)}{2}$

Note that $\alpha_{P L R}$ is determined solely by the working temperatures $\left(T_{H}\right.$ and $\left.T_{C}\right)$ and thermodynamic properties of the feed and permeate streams. Eq. (34) is a necessary but insufficient condition; the other condition needed for operation to reach PLR is fast (unlimited) mass transfer so that the limiting regime can be reached (the quantification of the mass transfer kinetics will be given in Section 4.2.3). Unlimited mass transfer kinetics can be attained using membranes with relatively high permeability or modules with large membrane area such that mass transfer is not limiting the module from attaining PLR.

The mass recovery rate in the PLR is given by

$\gamma_{P L R} \approx \alpha \frac{T_{H}^{*}-T_{C}}{\left(\bar{h}_{v a p, P} / c_{p, P}\right)-\left(\left(T_{H}^{*}-T_{C}\right) / 2\right)}$

which is directly proportional to the relative flow rate, $\alpha$. All other terms in Eq. (36) are constants that depend on the working temperatures and the thermodynamic properties of the feed and permeate streams. The mass recovery rate obtained from this analytical expression as a function of $\alpha$ is shown in Fig. 3A (dotted line). We note that $\gamma_{P L R}$ represents the theoretical maximum recovery achievable by DCMD when the relative flow rate is below the $\alpha_{P L R}$. If $\alpha$ is below the critical value $\alpha_{P L R}$, but mass transfer is limited due to insufficient membrane area or inadequately permeable membranes, the recovery rate will always be lower than $\gamma_{P L R}$.

\subsubsection{Feed limiting regime (FLR)}

Analogous to the case of PLR, the DCMD operation is in the FLR only if the relative flow rate $\alpha$ is higher than critical relative flow rate, $\alpha_{F L R}$, which is determined solely by the working temperatures and thermodynamic properties of the feed and permeate streams (Appendix C):

$\alpha \geq \alpha_{F L R}=\frac{T_{H}-T_{C}^{*}}{T_{H}^{*}-T_{C}} \frac{\left(\bar{h}_{v a p, F} / c_{p, P}\right)+\left(\left(T_{H}+T_{C}^{*}\right) / 2\right)-T_{H}^{*}}{\left(\bar{h}_{v a p, F} / c_{p, F}\right)+\left(c_{p, P} / c_{p, F}\right)\left(\left(T_{H}+T_{C}^{*}\right) / 2\right)-T_{C}^{*}}$

where $\bar{h}_{v a p, F}$ is the average enthalpy of vaporization of the feed and can be approximated by

$\bar{h}_{\text {vap }, F} \approx \frac{h_{\text {vap }}\left(C, T_{C}^{*}\right)+h_{\text {vap }}\left(C, T_{H}\right)}{2}$

Likewise, Eq. (37) is a necessary but not sufficient condition for FLR operation; the other condition requires the mass transfer to be unlimited so that the thermodynamic limiting regime can be reached within the defined module length.

The mass recovery rate in the FLR, $\gamma_{F L R}$, is given by

$\gamma_{F L R} \approx \frac{T_{H}-T_{C}^{*}}{\left(\bar{h}_{v a p, F} / c_{p, F}\right)+\left(c_{p, P} / c_{p, F}\right)\left(\left(T_{H}+T_{C}^{*}\right) / 2\right)-T_{C}^{*}}$

which is independent of the relative flow rate $\alpha$ (Fig. 3A, dashed horizontal line). Again, $\gamma_{F L R}$ signifies the theoretical maximum recovery achievable when $\alpha$ is greater than the critical value. If the relative flow rate is above $\alpha_{F L R}$, but mass transfer across the membrane is limiting, the recovery rate will be less than $\gamma_{F L R}$. Regardless of the feed and permeate (distillate) stream properties (i.e., temperatures and salt concentration) and the mass transfer kinetics, the mass recovery rate is always higher in FLR than in PLR. The limiting $\gamma_{F L R}$ signifies the theoretical maximum recovery achievable. It should also be noted that for a given temperature of the heat sink $\left(T_{C}\right), \gamma_{F L R}$ is practically proportional to the temperature of the heat source, $T_{H}$ (Fig. 3B). That is, for the example of DCMD desalination, increasing the working 
A

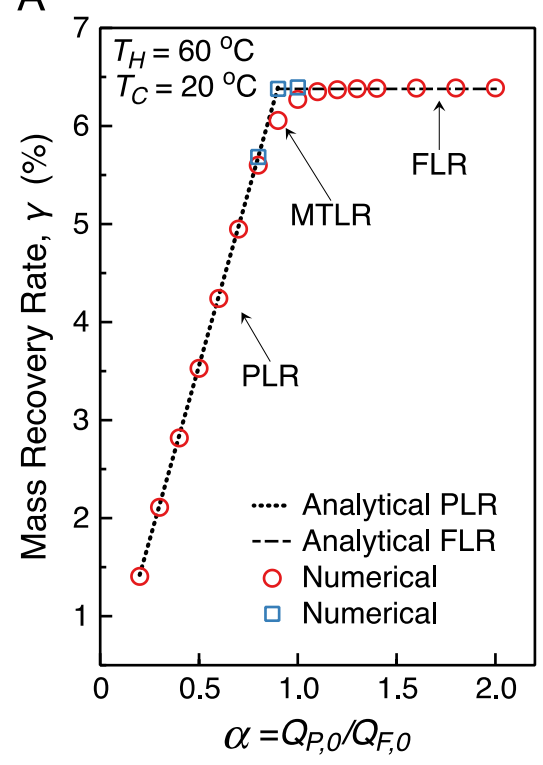

$\mathrm{B}$

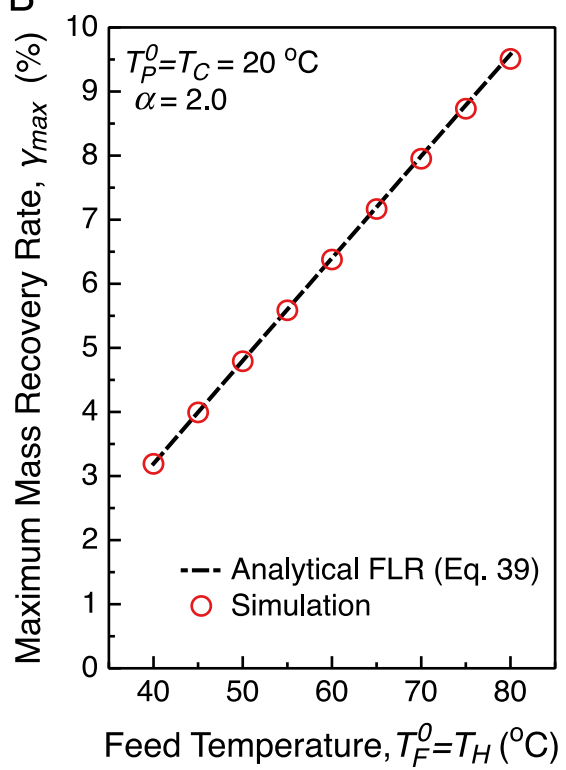

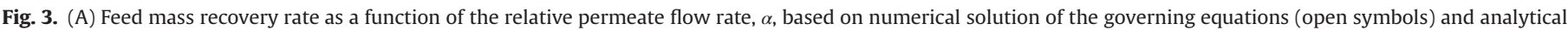

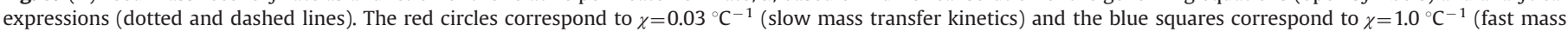

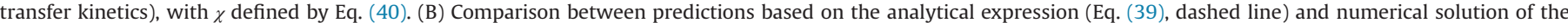

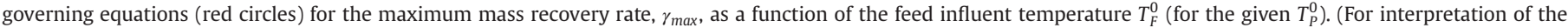
references to color in this figure legend, the reader is referred to the web version of this article.)

temperature of the influent feed stream leads to a corresponding linear increase in the mass recovery rate in a single pass.

\subsubsection{Mass transfer limiting regime (MTLR)}

The mass recovery rate, $\gamma$, as a function of the relative flow rate, $\alpha$, as obtained from numerical solution of the governing equations presented in Section 3 is shown in Fig. $3 \mathrm{~A}$ as red circles. The analytical expressions for predicting the mass recovery rate (Eqs. (36) and (39)) agree very well with the numerical solutions of the governing mass transfer equations, except for the case when $\alpha$ is very close to $\alpha_{F L R}$ or $\alpha_{P L R}$. This discrepancy occurs because when $\alpha$ is close to $\alpha_{F L R}$ or $\alpha_{P L R}$, mass transfer is limited with the same given MD module and flow rates, and the condition for either the PLR $\left(T_{P}^{f}=T_{H}^{*}\right)$ or the FLR $\left(T_{F}^{f}=T_{C}^{*}\right)$ cannot be met within a module of the given properties and flow rates described in the caption of Fig. 2. In this case, the DCMD system is operating in the MTLR.

Unlike the thermodynamically defined PLR and the FLR, the MTLR is kinetically defined. Strictly speaking, there exists no $\alpha$ that corresponds to MTLR, and although the analytical expressions for $\alpha_{P L R}$ (Eq. (34)) and $\alpha_{F L R}$ (Eq. (37)) have different mathematical forms due to the approximations used in the derivation, the calculated values of $\alpha_{P L R}$ and $\alpha_{F L R}$ under reasonable thermodynamic conditions in DCMD (i.e. $T_{H}, T_{C}$, and $C$ ) are numerically indistinguishable (Appendix C). Therefore, thermodynamically the DCMD operation is either permeate limited or feed limited. The MTLR occurs because the mass transfer is limited by either not having a high enough mass transfer coefficient or not having large enough membrane area. Therefore, MTLR signifies a kinetically constrained mode of operation in which the mass recovery rate is always smaller than the theoretical maximum achievable.

To quantify the degree to which mass transfer is limited, we define a parameter $\chi$ :

$\chi=\frac{K_{m, M D} A_{M D}}{Q_{F}^{0}}$

For a given feed flow rate, $Q_{F}^{0}$, a higher mass transfer coefficient, $K_{m, M D}$, or a larger membrane area, $A_{M D}$, both lead to a larger $\chi$ that is representative of faster mass transfer. It can be shown that as long as $\chi$ is kept constant, the temperature distribution profiles will be the same (Appendix D).

The above argument can further be confirmed by comparing the analytical expressions to the numerical results of the governing equations with much faster mass transfer kinetics as characterized by $\chi=1.0{ }^{\circ} \mathrm{C}^{-1}$ (blue squares in Fig. 3A), compared with the much lower $\chi=0.03{ }^{\circ} \mathrm{C}^{-1}$ (red circles in Fig. 3A). The numerical results fit the analytical predictions almost perfectly with fast mass transfer kinetics, implying that the analytical expressions for the mass recovery rate are consistent with the numerical simulations as long as the mass transfer is unlimited. Note that the lower the $\chi$ value is, the larger the range of $\alpha$ in which MTLR occurs. Therefore, whether an operation is limited by mass transfer or not is determined by both the mass transfer kinetics, $\chi$, and the relative flow rate, $\alpha$.

The limiting regimes, dictated by the temperature and solution properties of the feed and permeate streams, impose a thermodynamic limit on the maximum mass recovery rates attainable in DCMD, regardless of the membrane area or permeability. The mass transfer kinetics, quantified by the parameter $\chi$, can serve as a useful design parameter for DCMD systems to optimize membrane utilization and to ensure the separation process is not hindered by inadequate mass transfer. Therefore, to achieve high recoveries close to the thermodynamic limit in, for example DCMD desalination, sufficiently large membrane area or membrane permeability, relative to the feed flow rate, needs to be provided.

\subsection{Energy efficiency with heat recovery}

In the preceding section, we presented the analytical and numerical analyses of the DCMD module alone, from which we can calculate the mass recovery rate, $\gamma$. However, to quantify the energy efficiency of the DCMD process with heat recovery and to estimate the limits of heat recovery that can be achieved, it is necessary to conduct a system level investigation on the combined DCMD-HX (or simply MD-HX) system. 


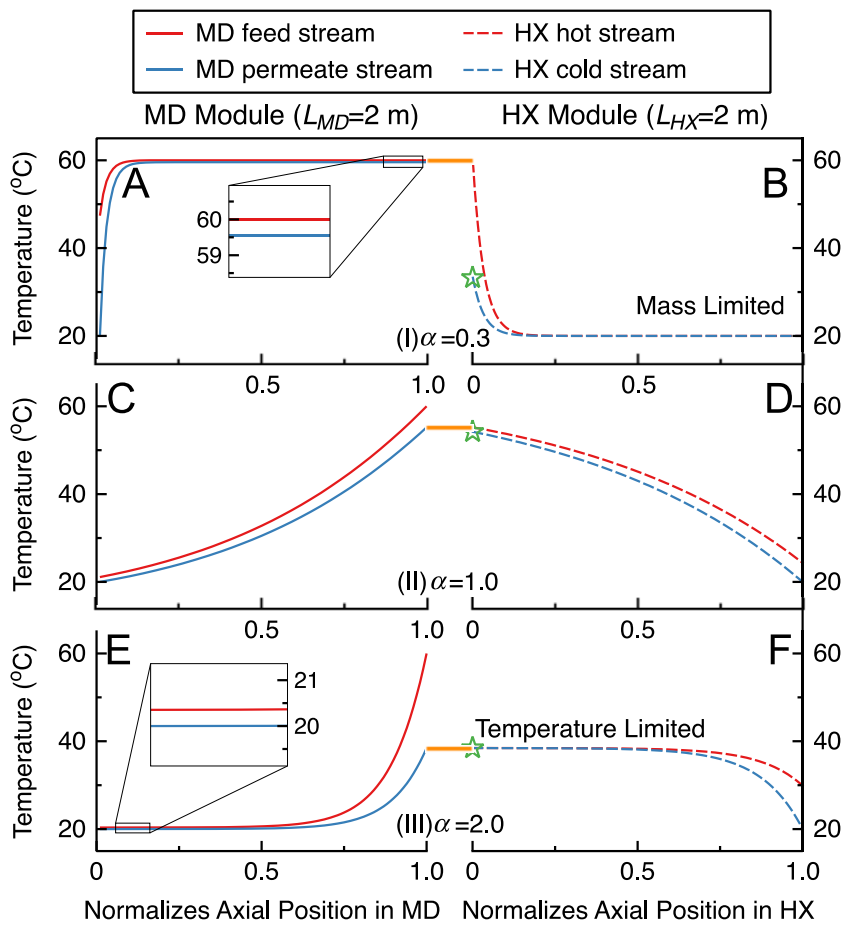

Fig. 4. Temperature distribution profiles of the feed (red solid line) and permeate (blue solid line) in the MD module $\left(T_{2}-T_{3}\right.$ and $T_{8}-T_{4}$, respectively, in Fig. $\left.1 \mathrm{~A}\right)$ and the hot stream (red dashed line) and cold stream (blue dashed line) in the HX module $\left(T_{4}-T_{5}\right.$ and $T_{0}-T_{1}$, respectively, in Fig. $\left.1 \mathrm{~A}\right)$ for different relative flow rates in the MD module: ( $\mathrm{A}$ and B) $\alpha=0.3$, ( $\mathrm{C}$ and $\mathrm{D}$ ) $\alpha=1.0$, and (E and F) $\alpha=2.0$. Orange line connects the blue solid curve and the red dashed curve to indicate that the effluent temperature of the MD permeate $\left(T_{4}\right.$ in Fig. $1 \mathrm{~A}$ ) is equal to the influent temperature of the HX module hot stream. The green star represents the effluent temperature of the cold stream in the HX ( $T_{1}$ in Fig. 1A), which is desirable to be high. Note that for the cases of PLR (A) and FLR (E) in the MD module, the feed and the permeate do not reach the same temperature because no mass transfer can occur when the temperature difference reaches $\Delta T_{t h}$. The insets in $\mathrm{A}$ and $\mathrm{E}$ zoom in on the temperatures of the feed and permeate streams at the location in the MD module where no mass transfer occurs. (For interpretation of the references to color in this figure legend, the reader is referred to the web version of this article.)

\subsubsection{Limiting regimes in heat recovery}

The mathematical coupling between the MD and HX modules is based on the description of the MD-HX system given in Section 2.1. The effluent temperature $T_{P}^{f}$ (or $T_{4}$ in Fig. $1 \mathrm{~A}$ ) and effluent flow rate $Q_{P}^{f}$ (or $Q_{4}$ ) of the permeate (distillate) in the MD module are the influent temperature $T_{h}^{0}$ and influent flow rate $Q_{h}^{0}$ of the hot stream in the HX module, respectively. Improving the energy efficiency of the overall operation requires the minimization of the energy input from the heat source $q_{H}$, which, for a given feed flow rate, is equivalent to maximizing the effluent temperature of the cold stream $T_{c}^{f}$ (or $T_{1}$ in Fig. 1A) in the HX module.

Fig. 4 shows the temperature profiles of the streams in the MD (left, solid lines) and HX (right, dashed lines) modules, for relative flow rates in the MD module of $\alpha=0.3,1.0$, and 2.0. Note that the relative flow rate in the HX module is dependent on $\alpha$ and the mass recovery rate, $\gamma$, in the MD module $\left(Q_{h}^{0} / Q_{c}^{0}=\alpha+\gamma\right)$. In the HX module, for a given influent flow rate of the cold stream $Q_{c}^{0}\left(Q_{0}\right.$ in Fig. 1A), a higher $T_{c}^{f}$ is equivalent to a higher effluent enthalpy of the HX cold stream, which entails a higher influent enthalpy of the hot stream $\left(\mathrm{S}_{4}\right.$ in Fig. 1A). As Fig. 4 shows, for an MD-HX system of given properties, $T_{c}^{f}$ (denoted by the green star symbols) is dependent on the relative flow rate $\alpha$.

At low $\alpha$ (e.g., $\alpha=0.3$, Fig. 4A and B), the MD operation is in the PLR. The influent temperature of the hot stream $T_{h}^{0}$ entering the HX module is high $\left(=T_{H}^{*}\right)$. However, because in this case the flow rate of the hot stream is relatively small compared to that of the
Table 1

Summary of the performance parameters at the different $\alpha$ values used in Fig. 4.

\begin{tabular}{lccc}
\hline$\left(\boldsymbol{\alpha}=\boldsymbol{Q}_{\boldsymbol{P}}^{\mathbf{0}} / \boldsymbol{Q}_{\boldsymbol{F}}^{\mathbf{0}}\right)$ & $\mathbf{0 . 3}$ & $\mathbf{1 . 0}$ & $\mathbf{2 . 0}$ \\
\hline Mass recovery rate, $\gamma(\%)$ & 2.11 & 6.27 & 6.39 \\
Specific heat duty, $\beta\left(\mathrm{kJ} \mathrm{kg}^{-1}\right)$ & 5080.1 & 371.2 & 1351.2 \\
Heat recovery efficiency, $\lambda(\%)$ & 33.1 & 85.5 & 46.1 \\
\hline
\end{tabular}

cold stream (the ratio is $\alpha+\gamma$ ), the total heat energy or enthalpy that can be transferred over from the hot stream is low despite the higher starting temperature, $T_{h}^{0}$. This is clearly illustrated in Fig. 4B, where, due to the relatively low relative flow rate $(\alpha+\gamma)$, the hot stream cools down rapidly in the HX module without transferring enough heat to the cold stream for its temperature to reach $T_{h}^{0}$. We classify this scenario as mass-limited heat recovery.

On the other hand, if $\alpha$ is high (e.g., $\alpha=2.0$, Fig. 4E and F), the effluent temperature of the MD permeate, $T_{P}^{f}$, and, correspondingly, the influent temperature of the HX hot stream, $T_{h}^{0}$, are relatively low because the MD operates in the FLR. Therefore, even though the effluent temperature of the cold stream, $T_{c}^{f}$, can reach $T_{h}^{0}$ in the HX module (Fig. $4 \mathrm{~F}$ ), $T_{c}^{f}$ is still considerably lower than the specified working temperature $T_{H}$ because the MD feed influent as $T_{h}^{0}\left(=T_{P}^{f}\right)$ is low. The heat recovery in this case is limited by $T_{h}^{0}\left(=T_{P}^{f}\right)$, which is the highest temperature $T_{c}^{f}$ can ever reach. We classify this scenario as temperature-limited heat recovery.

Both the mass- and temperature-limited heat recovery scenarios represent inefficient energy recovery for the overall MD-HX system. As the effluent temperature of the HX cold stream (which is channeled to the MD module as the influent feed stream) is relatively low, considerable amount of energy needs to be supplied to bring $T_{c}^{f}$ to the specified MD working temperature. Only in an optimal range of $\alpha$ is the operation neither mass- nor temperaturelimited (e.g., $\alpha=1.0$, Fig. 4C and D). In this case, the highest $T_{c}^{f}$ and correspondingly the lowest required $q_{H}$, of the three scenarios, can be attained.

\subsubsection{Overall energy efficiency and optimal operation condition}

The process performance parameters and energy efficiency for the three scenarios in Fig. $4(\alpha=0.3,1.0$, and 2.0) are summarized in Table 1. Consistent with the analysis shown in Fig. 3A, the mass recovery rate $\gamma$ increases with increasing $\alpha$. The specific heat duty $\beta$ (Eq. (26), i.e., energy input required to produce a unit mass of pure water) is, however, found to be the lowest among the three scenarios at $\alpha=1.0$. Similarly, the efficiency of heat recovery, $\lambda$ (Eq. (29), i.e., fraction of heat recovered in the HX compared to the total energy in bringing the feed stream temperature from that of the heat sink to that of the heat source), is the highest among the three scenarios for $\alpha=1$. Note that $\beta$ is not simply negatively correlated to $\lambda$, as Eq. (30) shows that $\gamma$ also plays an important role in determining $\beta$.

Notably, at $\alpha=0.3$, the specific heat duty is even higher than the enthalpy of vaporization (i.e. $\sim 2400 \mathrm{~kJ} \mathrm{~kg}^{-1}$ ), which implies that using an MD-HX system for desalination under such a condition is even more energy consuming than simply evaporating the feed water in a batch mode without any heat recovery. The reason for such inefficiency is straightforward: a portion of the available heat energy in the MD feed stream is not transferred to the MD permeate stream when the system operates in the PLR. This unutilized portion of enthalpy becomes particularly significant when $\alpha$ is small. In the example presented in Fig. 4A, the effluent temperature of the MD feed $T_{F}^{f}$ is still quite high $\left(\sim 50{ }^{\circ} \mathrm{C}\right)$. With the design shown in Fig. 1A, this energy embedded in the warm MD feed effluent (stream $S_{3}$ ) is discharged out of the system 
A

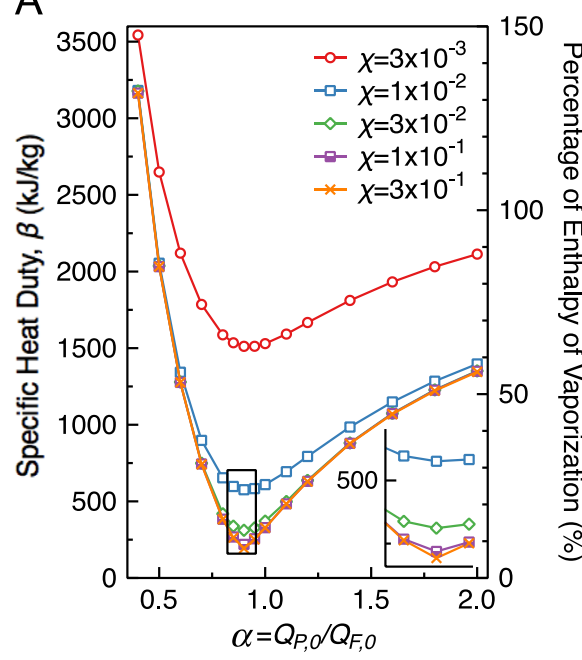

B

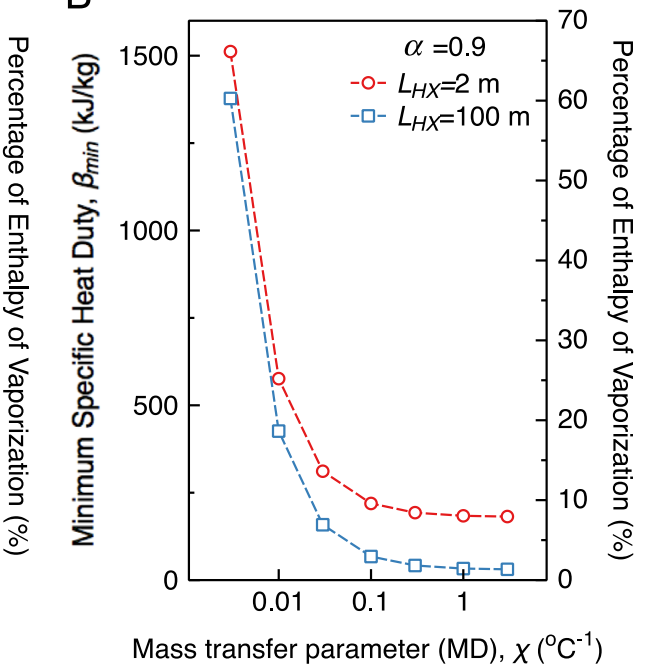

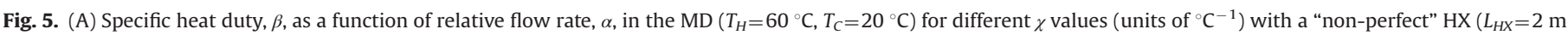

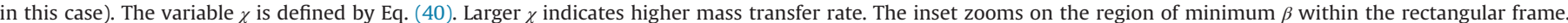

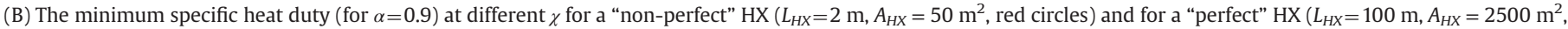

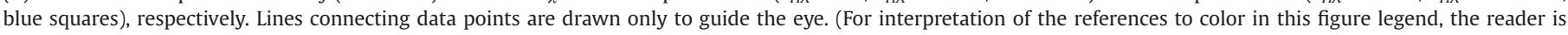
referred to the web version of this article.)

without being used. Therefore, an MD-HX operation with low $\alpha$ may require energy input in excess of the enthalpy of vaporization to carry out the separation.

The above analysis of the three representative scenarios ( $\alpha=0.3,1.0$, and 2.0) suggests that there exists an optimal $\alpha$ where the MD-HX system is most energy efficient. To substantiate the existence of such an optimal $\alpha$, we expanded the matrix of the numerical simulation to cover a broader range of $\chi$ (representing mass transfer kinetics as defined by Eq. (40)) and a more refined range of the relative flow rate in the MD module, $\alpha$. The calculated specific heat duties, $\beta$, as a function of the relative flow rate $\alpha$ are presented in Fig. 5A. It is observed that, for all $\chi$ values investigated, $\beta$ is minimum when $\alpha$ is around 0.9 (the resolution of $\alpha$ for the simulation was 0.05 ), which is close to the critical $\alpha$ that divides the PLR and FLR as presented in Sections 4.2.1 and 4.2.2. The critical $\alpha$ calculated from the analytical expressions, Eq. (34) $\left(\alpha_{P L R}\right)$ or $(37)\left(\alpha_{F L R}\right)$, is 0.918 , remarkably close to the optimal $\alpha$ obtained from the numerical simulations shown in Fig. 5A.

The theoretical basis for this apparent coincidence can be elucidated from the expression for $\beta$ (Eq. (28)). The specific heat duty can be minimized by increasing $T_{1}$, which is the effluent temperature of the HX cold stream, $T_{c}^{f}$. For a given HX, a higher $T_{c}^{f}$ can be attained with a higher HX hot stream temperature, $T_{h}^{0}$, which is equal to the effluent temperature of the MD permeate, $T_{P}^{f}$. The highest $T_{P}^{f}$ attainable is $T_{H}^{*}$ (Eq. (32)), which occurs only if the MD operation is in PLR, thus requiring $\alpha \leq \alpha_{P L R}$ (Eq. (34)). Alternatively, $\beta$ can be minimized by, according to Eq. (28), increasing the mass recovery rate, $\gamma$. As discussed earlier in Section 4.2.2 and shown in Fig. 3A, $\gamma$ reaches maximum when the MD operates in FLR, which requires that $\alpha \geq \alpha_{F L R}$ (Eq. (37)). Because $\alpha_{F L R}$ and $\alpha_{P L R}$ are practically identical (Section 4.2.3), we collectively term them the critical relative flow rate, $\alpha^{*}$, hereafter. Operating the MD-HX system at $\alpha^{*}$, simultaneously satisfies both conditions for maximizing $T_{1}$ and $\gamma$, thus resulting in the overall minimum in the specific heat duty.

We note that the general idea about the existence of an optimal relative flow rate is also supported by simulation results for DCMD operated in a cascade of cross-flow hollow fiber module with heat recovery, as reported by Lee et al. [48]. In that study, even though the operation mode was not in counter-current flow as in our paper, the simulation results also showed an optimal relative flow rate close to one that corresponds to a maximum gain output ratio (or performance ratio).

The critical relative flow rate can be determined using either Eq. (34) or (37) for a feed solution of given concentration and a set of working temperatures $\left(T_{H}\right.$ and $\left.T_{C}\right)$, and it is not dependent on the kinetics of mass transfer in the MD module or the heat transfer in HX. The existence of an optimal $\alpha$ that is independent of the mass and heat transfer kinetics is very important, as it provides the basis for $\alpha^{*}$, which can be calculated solely based on thermodynamics, to guide an actual MD-HX operation to achieve its highest energy efficiency, regardless of the mass and heat transfer kinetics of the system.

The specific heat duty $\beta$ is apparently affected by the kinetics of mass transfer in the MD and heat transfer in the HX, even though $\alpha^{*}$ at which $\beta$ reaches minimum is not. As Fig. 5A shows, for any given $\alpha$, increasing the mass transfer kinetics parameter $\chi$ causes $\beta$ to asymptotically approach a limit that corresponds to unlimited mass transfer. This trend is more clearly demonstrated by the red circles in Fig. 5B, which shows $\beta$ as a function of $\chi$ for $L_{H X}=2 \mathrm{~m}$ $\left(A_{H X}=50 \mathrm{~m}^{2}\right)$, demonstrating a drastic initial reduction in $\beta_{\min }$ that gradually levels offs as $\chi$ increases from 0.003 to 3 . When $\chi=0.03$, a reasonable value in practice, $\beta_{\min }$ is already fairly close to its lowest limit.

The $\beta$ values presented in Fig. 5A, and as the red circles in Fig. 5B, were obtained using an $\mathrm{HX}$ with finite heat transfer kinetics, which manifests as a non-zero difference between the temperatures of the hot and the cold streams of the HX, $T_{h}^{0}$ and $T_{c}^{f}$, respectively. We refer to an HX with finite heat transfer kinetics as a "non-perfect" HX. This difference in temperature can become infinitesimal if the heat transfer kinetics in the HX are greatly enhanced by, for example, increasing the contact area for the heat exchange. The blue squares in Fig. 5B show $\beta_{\min }$ as a function of $\chi$ when $\alpha=0.9$, with $L_{H X}=100 \mathrm{~m}\left(A_{H X}=2500 \mathrm{~m}^{2}\right.$, i.e., the heat exchange area is increased 50 times compared to the red circles), which results in negligible difference between $T_{h}^{0}$ and $T_{c}^{f}$ in the HX. We refer to such an HX as a "perfect" HX. With such enhanced HX heat transfer kinetics, the lower limit of $\beta$ at $\alpha=0.9$ is reduced to about $31 \mathrm{~kJ} \mathrm{~kg}^{-1}$, which is very close to the ultimate thermodynamic limit (i.e. the lowest $\beta_{\min }$ that is theoretically achievable) 
of $27.6 \mathrm{~kJ} \mathrm{~kg}^{-1}$ calculated using Eq. (28) with $\alpha=\alpha^{*}=0.918, \gamma=\gamma_{F L R}$, and $T_{1}=T_{H}^{*}$. At the ultimate thermodynamic limit, the minimum specific heat duty is less than $1.2 \%$ of the enthalpy of vaporization, leading to a performance ratio (PR, Eq. (27)) of $\sim 84$. The ultimate thermodynamic limit of $\beta_{\min }$, which occurs at $\alpha^{*}$ with unlimited mass transfer in MD and unlimited heat transfer in HX, can be easily calculated using this approach for different operating conditions (i.e., feed concentration and working temperature).

It is worthwhile to note that the calculated thermodynamic limit of the specific heat duty $\beta$, or of the PR, is only theoretically achievable as a result of the many idealities assumed in the derivation. In addition, it is also not economically preferable to pursue the highest thermodynamic efficiency, as it requires very large area for the MD membrane and heat exchanger, which results in low production rate per area of membrane/heat exchanger and, consequentially, unreasonably high capital cost for a target treatment capacity. Regardless, as mentioned before, it is always preferable to operate the MD-HX system at the critical relative flow rate $\alpha^{*}$, as it always yields the highest energy efficiency for a given system (Fig. 5A).

\subsection{Impact of conductive heat transfer on MD performance}

The discussion up to this point assumes an extremely insulating membrane matrix such that $B \rightarrow \infty$ ( $B$ defined by Eq. (14)) and, hence, the conductive heat transfer at the feed-membrane interface $\zeta_{F}$ (defined by Eq. (16)) approaches zero. In reality, neither the membrane is infinitely thick, nor the the thermal conductivity of the membrane matrix is infinitely large, and therefore the parameter $B$ is finite. In this section, we account for the effects of conductive heat transfer on the MD performance by considering a thermally-conductive membrane.

We define a conductive heat transfer coefficient for the MD membrane, $K_{c, M D}$, as the ratio of thermal conductivity, $k_{c, M D}$, over
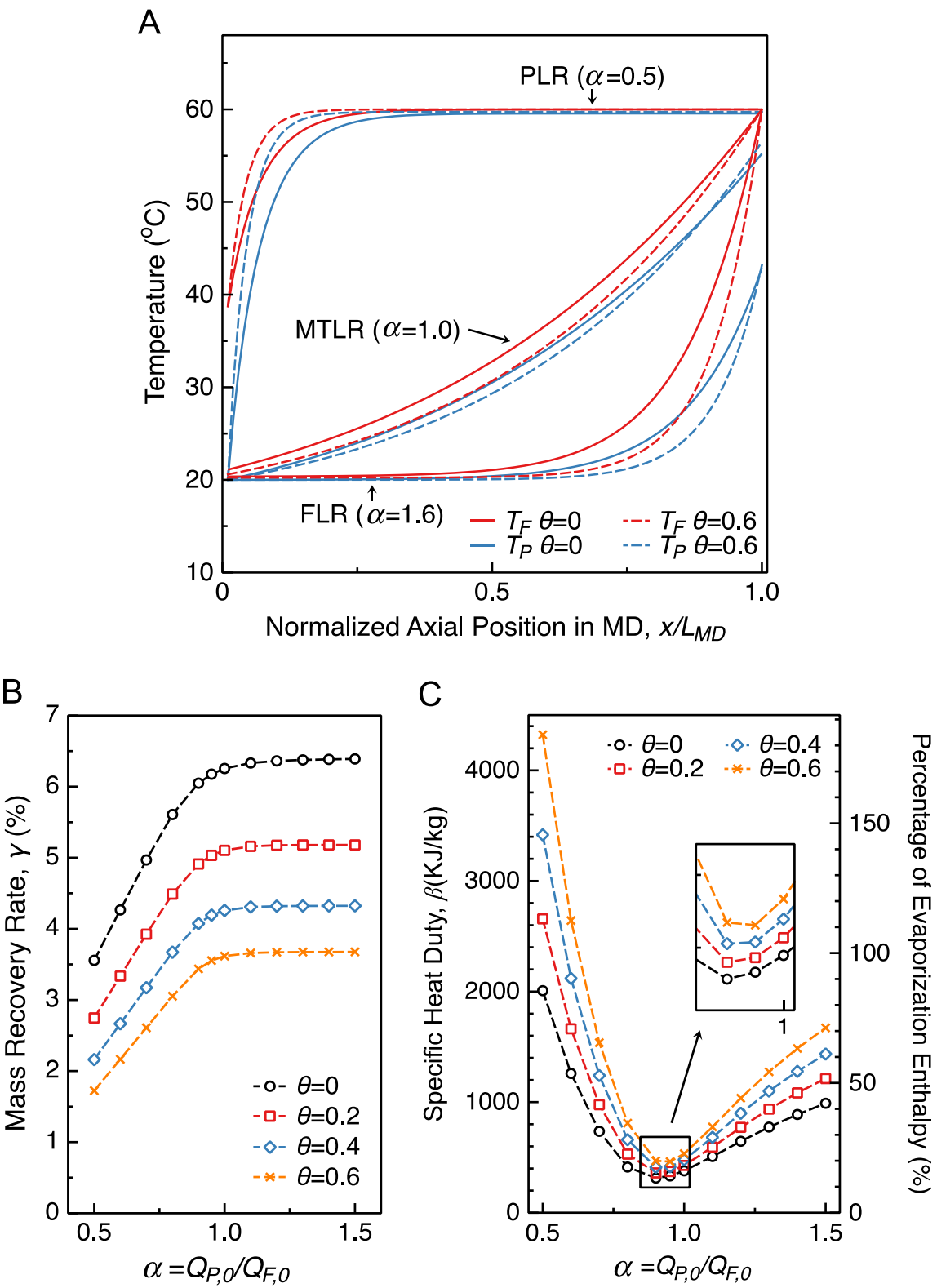

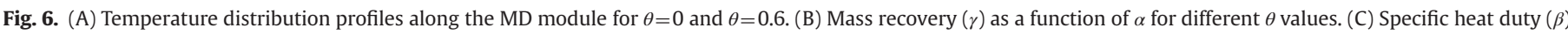

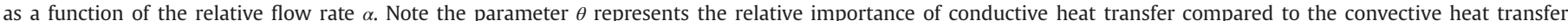
(defined by Eq. (43)). For all calculations, $A_{M D}=50 \mathrm{~m}^{2}, K_{m, M D}=0.03 \mathrm{~kg} \mathrm{~m}^{-2} \mathrm{~K}^{-1} \mathrm{~min}^{-1}$, and $Q_{F}^{0}=50 \mathrm{~kg} \mathrm{~min}^{-1}$. For $(\mathrm{C}), A_{H X}=50 \mathrm{~m}^{2}$, and $K_{c, H X}=10^{3} \mathrm{~W} \mathrm{~m}^{-2}{ }^{\circ} \mathrm{C}$. 
the thickness of the membrane, $\delta$ :

$K_{c, M D}=\frac{k_{c, M D}}{\delta}$

The typical conductivity of the solid material (polymer) of the membrane $\left(k_{c, S}\right)$ ranges from 0.1 to $0.3 \mathrm{~W} \mathrm{~m}^{-1}{ }^{\circ} \mathrm{C}^{-1}$, whereas the typical conductivity of air/vapor $\left(k_{c, V}\right)$ ranges from 0.02 to $0.03 \mathrm{~W} \mathrm{~m}^{-1}{ }^{\circ} \mathrm{C}^{-1}$ [20]. Assuming a porosity of $\varepsilon=0.8$ [15], $k_{c}$ can be estimated using the following equation [17]:

$k_{c, M D}=(1-\varepsilon) k_{c, S}+\varepsilon k_{c, V}$

Using the above values for $k_{c, S}$ and $k_{c, V}$ along with Eq. (42), the range of $k_{c, M D}$ is estimated to be from 0.04 to $0.08 \mathrm{~W} \mathrm{~m}^{-1}{ }^{\circ} \mathrm{C}^{-1}$. A typical MD membrane thickness is about $10^{-4} \mathrm{~m}$ [7]. Therefore, a typical range of $K_{c, M D}$ is calculated to be between 400 and $800 \mathrm{~W} \mathrm{~m}^{-2}{ }^{\circ} \mathrm{C}^{-1}$, which is consistent with typical values reported in literature $[11,17]$.

To compare the relative importance of the conductive heat transfer and the convective heat transfer (i.e., heat carried by the vapor flux), we define a dimensionless parameter $\theta$ :

$\theta=\frac{K_{c, M D}}{h_{v a p} K_{m, M D}}=\frac{k_{c, M D} / \delta}{h_{v a p} K_{m, M D}}$

If we fix the membrane mass transfer coefficient $K_{m, M D}$ to be $0.03 \mathrm{~kg} \mathrm{~m}^{-2} \mathrm{~min}^{-1}{ }^{\circ} \mathrm{C}^{-1}$ and use an approximate value of $2400 \mathrm{~kJ} \mathrm{~kg}^{-1}$ for $h_{\text {vap }}, \theta$ will range between 0.3 and 0.6 , with a larger $\theta$ signifying greater effect of conductive heat transfer compared to convective heat transfer.

Fig. 6A shows the comparison between the MD temperature distribution profiles with $\theta=0$ (i.e., minimal conductive heat transfer by the membrane) and those with $\theta=0.6$ for the three different operation regimes (i.e., PLR, FLR, and MTLR). In general, conductive heat transfer enhances the overall heat transfer kinetics. For operations in the PLR and FLR, enhanced conductive heat transfer reduces the effective length of MD where mass transfer occurs, but changes in the MD feed and permeate effluent temperatures are almost negligible. When the system operates in PLR (FLR), the change of temperature in the feed (permeate) stream is constant (from $T_{C}$ to $T_{H}^{*}$ for the permeate in PLR, and from $T_{H}$ to $T_{C}^{*}$ for the feed in FLR), which means that the heat transferred to (from) the permeate (feed) stream should be approximately the same regardless of the heat transfer mechanism. However, unlike the behavior for the PLR and FLR, conductive heat transfer influences the effluent temperatures for operation in the MTLR, though not to a large extent.

Fig. $6 \mathrm{~B}$ shows the mass recovery, $\gamma$, as a function of the relative permeate mass flow rate, $\alpha$, for different values of the dimensionless conductive heat transfer parameter, $\theta$. The mass recovery rate, decreases monotonically as $\theta$ increases across the range of $\alpha$. Therefore, higher conductive heat transfer is increasingly detrimental to the performance of DCMD. The reduced $\gamma$ is attributed to the loss of part of the driving force for mass transfer through conductive heat transfer.

Fig. $6 \mathrm{C}$ shows the specific heat duty, $\beta$, as a function of relative flow rate, $\alpha$, for different values of the dimensionless conductive heat transfer parameter $\theta$. Another consequence of conductive heat transfer is the increased specific heat duty $\beta$-that is, more energy is requried to generate a unit mass of product water compared to the ideal case with minimal conductive heat transfer (i.e., when $\theta=0$ ). The increased specific heat duty is attributed to the consumption of a portion of the supplied heat energy to compensate for the conductive heat loss, which does not contribute to mass transfer. Therefore, mitigating conductive heat transfer via optimal membrane design to reduce the conductive heat transfer coefficient is of great importance in achieving an energy efficient DCMD process.

\section{Concluding remarks}

In an actual MD separation process, the extent of the separation (i.e., the recovery rate) and the energy requirement (i.e., the specific heat duty) will be key performance parameters. Both the numerical simulation and the theoretical thermodynamic analysis indicate that the mass recovery rate increases proportionally with increasing the relative mass flow rate between the permeate and the feed $(\alpha)$ in the permeate limiting regime (PLR), and eventually becomes constant with respect to $\alpha$ in the feed limiting regime (FLR). This signifies the existence of a thermodynamic limit on the maximum mass recovery $\left(\gamma_{\max }\right)$ that is determined solely by the thermodynamic properties of the feed and permeate streams, and the working temperatures.

The results of our modeling and thermodynamic analysis have important implications for MD separation applications. For example, seawater desalination in a single-pass $\mathrm{MD}$ at $T_{H}=60{ }^{\circ} \mathrm{C}$ can never achieve a feed water recovery rate higher than $6.4 \%$, regardless of the mass transfer kinetics. This is substantially below the typical recovery rate of $40-60 \%$ for seawater reverse osmosis desalination in a single pass. Therefore, to achieve higher recovery rates for cost-effective operation, it is necessary to recirculate the effluent of the MD feed stream (i.e. the brine) back to the influent of the heat exchanger (HX) cold stream.

Our analysis also demonstrates, quantitatively, that coupling an HX to MD can theoretically recover the majority of the latent heat accumulated in the permeate stream, thereby reducing the thermal energy input. The ultimate minimum specific heat duty $\beta_{\text {min }}^{*}$ (i.e., the minimum energy required to produce a unit mass of the distillate with unlimited mass transfer kinetics) is determined solely based on thermodynamics and represents the minimum energy of separation in a single-pass MD-HX. For seawater desalination by MD at $60^{\circ} \mathrm{C}$, this minimum energy is $27.6 \mathrm{~kJ} \mathrm{~kg}^{-1}$. Compared to the theoretical minimum energy consumption of reverse osmosis with a typical recovery of $50 \%\left(\sim 3.18 \mathrm{~kJ} \mathrm{~kg}^{-1}\right)$ [49], the minimum energy requirement for MD desalination is still relatively high. However, because reverse osmosis utilizes high cost electric energy, MD can be an attractive low-cost option when low-grade thermal energy is readily available. Additionally, our analysis shows that the minimum specific heat duty always occurs at the critical relative flow rate $\alpha^{*}$, regardless of the mass and heat transfer kinetics in the MD and HX modules. As such, to achieve the highest energy efficiency, a single-pass MD-HX should operate at the critical relative flow rate.

Finally, we also assessed the detrimental impact of conductive heat transfer through the MD membrane matrix on the system performance. Inevitable conductive heat transfer across an actual MD membrane causes parasitic loss in the driving force, and reduces both the mass recovery rate in the DCMD operation and the energy efficiency of the MD-HX system. However, increasing the thermal insulation of MD membranes by increasing thickness usually comes at the expense of reducing permeability. Therefore, in order to achieve high system performance, the challenge will be to develop DCMD membranes with relatively low heat conductivity, but still maintain reasonably high permeability.

\section{Acknowledgements}

We acknowledge the support received from the National Science Foundation under Award Number CBET 1232619 and from the Advanced Research Projects Agency-Energy (ARPAE), U.S. Department of Energy, via Grant DE-AR0000306. 
Appendix A. Correlation equations and representative curves for $h_{v a p}(C, T)$ and $c_{p, L}(C, T)$ of an $\mathrm{NaCl}$ solution

The specific enthalpy of vaporization $h_{v a p}(C, T)$ can be calculated from the Clausius-Clapeyron relation:

$h_{\text {vap }}(C, T)=R T^{2}\left(\frac{d \ln \left(P_{S}^{0}(T)\right)}{d T}+\frac{d \ln \left(a_{S}(C, T)\right)}{d T}\right)$

where $R$ is the ideal gas constant, $T$ is the absolute temperature, $P_{S}^{0}(T)$ is the equilibrium partial vapor pressure of the pure solvent (water in our case) at temperature $T$, and $a_{S}$ is the activity of the solvent in an $\mathrm{NaCl}$ solution of concentration $C$ at temperature $T$. A correlation equation has been proposed for calculating $\ln \left(P_{S}^{0}(T)\right)$ for pure water [42]:

$\ln \left(P_{S}^{0}(T)\right)=\ln \left(P_{c r t}\right)+\frac{T_{c r t}}{T}\left(a_{1} \tau+a_{2} \tau^{1.5}+a_{3} \tau^{3}+a_{4} \tau^{6}\right)$

where $P_{c r t}$ is the critical vapor pressure, $T_{c r t}$ is the critical temperature, and the coefficients $a_{i}$ are summarized in Table A1. The variable $\tau$ is defined as

$\tau=1-\frac{T}{T_{c r t}}$

Table A1

Parameters used in Eqs. (A1)-(A10) (for $\mathrm{NaCl}$ solution).

\begin{tabular}{lllc}
\hline Eq. (A2) & & \multicolumn{2}{l}{ Eqs. (A9) and (A10) } \\
\hline$T_{c r t}$ & $647.3 \mathrm{~K}$ & $k_{1}$ & $3.369 \times 10^{-1}$ \\
$P_{c r t}$ & $22.12 \mathrm{MPa}$ & $k_{2}$ & $-6.321 \times 10^{-4}$ \\
$a_{1}$ & -7.7645 & $k_{3}$ & 9.143 \\
$a_{2}$ & 1.4584 & $k_{4}$ & $-1.351 \times 10^{-2}$ \\
$a_{3}$ & -2.7758 & $k_{5}$ & $2.261 \times 10^{-3}$ \\
$a_{4}$ & -1.2330 & $k_{6}$ & $1.921 \times 10^{-6}$ \\
& & $k_{7}$ & $4.526 \times 10^{-1}$ \\
Eq. (A7) & & Eq. (A8) & \\
$b_{0}$ & 3.4048 & $S_{0}$ & $3.6984 \times 10$ \\
$b_{1}$ & $2.078 \times 10^{-2}$ & $S_{1}$ & $1.706 \times 10^{-1}$ \\
$b_{2}$ & $-9.878 \times 10^{-5}$ & $S_{2}$ & $-1.35 \times 10^{-3}$ \\
\hline
\end{tabular}

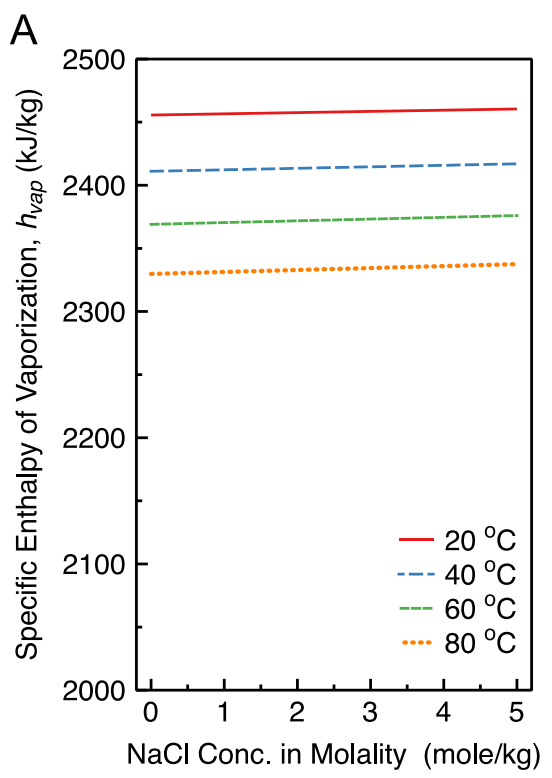

The derivative of $P_{S}^{0}(T)$ with respect to $T$ to be used in Eq. (A1) is

$$
\begin{aligned}
\frac{d \ln \left(P_{S}^{0}(T)\right)}{d T}= & -\frac{T_{c r t}}{T^{2}}\left(a_{1} \tau+a_{2} \tau^{1.5}+a_{3} \tau^{3}+a_{4} \tau^{6}\right) \\
& -\frac{1}{T}\left(a_{1}+1.5 a_{2} \tau^{0.5}+3 a_{3} \tau^{2}+6 a_{4} \tau^{5}\right)
\end{aligned}
$$

The influence of salt concentration is reflected by the change of solvent activity. The natural-logarithm of solvent activity in the presence of electrolytes is given by

$\ln \left(a_{S}\right)=-v m M_{S} \phi$

where $v=v_{M}+v_{X}$, with $v_{M}$ and $v_{X}$ being the stoichiometric coefficients of the cation and anion of the electrolyte $\mathrm{MX}$ (e.g., for $\mathrm{NaCl}$, $v=2$ ), $m$ is the molality of the solution, $M_{S}$ is the molecular weight of the solvent, and $\phi$ is the osmotic coefficient of the solution. The latter can be calculated based on the theory of electrolyte solutions [41]:

$\phi=1-\left|z_{+} z_{-}\right| A_{\phi}\left(\frac{I^{1 / 2}}{I+b I^{1 / 2}}\right)+\frac{S}{T v}\left(\frac{2 n}{2 n+1}\right) I^{2 n}$

In Eq. (A6), $z_{+}$and $z_{-}$are the charges of the cation and anion of the electrolyte, respectively, and $I$ is the ionic strength of the solution. The three adjustable parameters in this equation, $b, S$, and $n$, can be calculated using correlation equations. However, Ge et al. have shown that $n$ can be treated as a constant $(n=0.645)$ without compromising the accuracy. The correlation equations for the adjustable parameters $b$ and $S$ as a function of $T$ are given as [41]

$b(T)=b_{0}+b_{1}(T-298.15 \mathrm{~K})+b_{2}(T-298.15)^{2}$

and

$S(T)=S_{0}+S_{1}(T-298.15 \mathrm{~K})+S_{2}(T-298.15)^{2}$

with the coefficients $b_{i}$ and $S_{i}$ given in Table A1 as well.

The remaining variable in Eq. (A6) is the Debye-Huckel parameter $A_{\phi}$ which can be calculated using the following correlation equation [42]:

$A_{\phi}=k_{1}+k_{2} T+\frac{k_{3}}{T}+k_{4} \ln (T)+\frac{k_{5}}{T-263 \mathrm{~K}}+k_{6} T^{2}+\frac{k_{7}}{680 \mathrm{~K}-T}$

The coefficients $k_{i}$ are also be given in Table A1. The derivative of

B

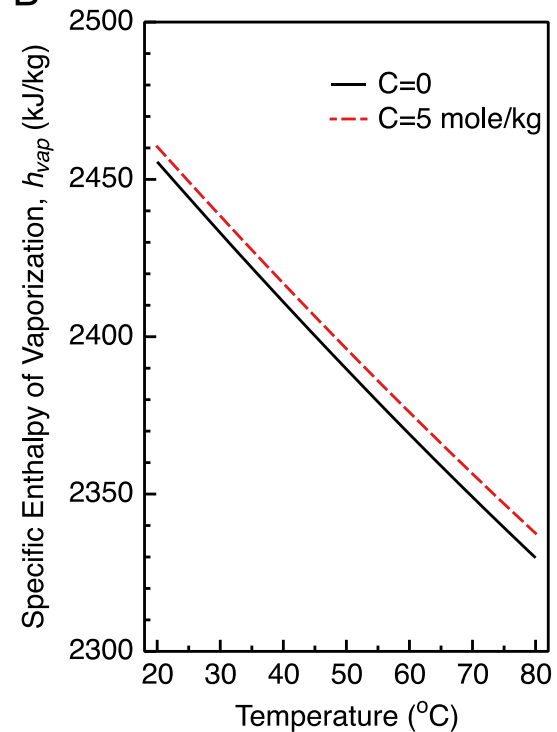

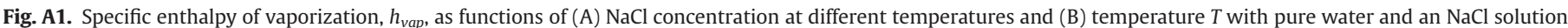
of $5 \mathrm{~mol} \mathrm{~kg}^{-1}$. 
$A_{\phi}$ with respect to $T$ is

$\frac{d A_{\phi}}{d T}=k_{2}-\frac{k_{3}}{T^{2}}+\frac{k_{4}}{T}-\frac{k_{5}}{(T-263 \mathrm{~K})^{2}}+2 k_{6} T+\frac{k_{7}}{(680 \mathrm{~K}-T)^{2}}$

Note that all temperatures used in the equations presented above are absolute temperatures (in units of Kelvin).

With Eqs. (A1)-(A10) and the coefficients given in Table A1, $h_{\text {vap }}$ can be numerically evaluated for any given $C$ and $T_{P}$. The dependences of $h_{\text {vap }}$ on $C$ and on $T_{P}$ are illustrated in Fig. A1 with representative curves.

The heat capacity of an aqueous $\mathrm{NaCl}$ solution, $c_{p}$, at different concentrations and temperatures can also be evaluated using reported correlation equations [43]:

$c_{p}=w_{W} c_{p, W}+w_{S} c_{p, S}$

where $c_{p, W}$ the heat capacity of pure water, $c_{p, S}$ is the heat capacity of the solute (i.e. $\mathrm{NaCl}$ ), and $w_{W}$ and $w_{S}$ are the mass fractions of pure water and solute, respectively.

The dependence of $c_{p, w}$ on temperature has been tabulated [42] and we propose the following correlation equation based on the tabulated data:

$c_{p, W}=c_{0}+c_{1} T+c_{2} T^{2}+c_{3} T^{3}+c_{4} T^{4}+c_{5} T^{5}$

The heat capacity of the solute $c_{p, S}$ can be calculated using the proposed correlation [42]:

$c_{p, S}=f_{1} \exp \left(f_{2} T+f_{3} \exp (0.01 T)+f_{4} w_{S}\right)+f_{5} w_{S}^{f_{6}}$

Note that the temperatures $T$ in both Eqs. (A12) and (A13) are in Celsius. The coefficients $c_{i}$ and $f_{i}$ are given in Table A2.

The dependences of $c_{p}$ on $C$ and on $T$ are illustrated in Fig. A2 with representative curves.

Table A2

Parameters used in Eqs. (A12) and (A13) (for $\mathrm{NaCl}$ solution).

\begin{tabular}{lrlc}
\hline Eq. (A12) & \multicolumn{3}{c}{ Eq. (A13) } \\
\hline$c_{0}$ & 4.22 & $f_{1}$ & $-6.94 \times 10^{-2}$ \\
$c_{1}$ & $-3.17 \times 10^{-3}$ & $f_{2}$ & $-7.82 \times 10^{-2}$ \\
$c_{2}$ & $9.87 \times 10^{-5}$ & $f_{3}$ & 3.85 \\
$c_{3}$ & $-1.59 \times 10^{-6}$ & $f_{4}$ & -11.28 \\
$c_{4}$ & $1.37 \times 10^{-8}$ & $f_{5}$ & 8.73 \\
$c_{5}$ & $-4.00 \times 10^{-11}$ & $f_{6}$ & 1.81 \\
\hline
\end{tabular}

\section{Appendix B. Correlation equation for $\Delta T_{t h}\left(C, T_{P}\right)$}

For any given $C$ and $T$, the threshold temperature difference can be calculated using the following equation:

$\Delta T_{\text {th }}\left(C, T_{P}\right)=T_{F}^{*}\left(C, T_{P}\right)-T_{P}$

with $T_{F}^{*}$ being defined such that

$p_{W}\left(C, T_{F}^{*}\right)=p_{W}\left(0, T_{P}\right)$

here $p_{W}\left(0, T_{P}\right)$ is the partial vapor pressure of pure water and can be calculated using Eq. (A2). To calculate $p_{W}\left(C, T_{F}^{*}\right)$, we should realize

$p_{W}\left(C, T_{F}^{*}\right)=p_{W}\left(0, T_{F}\right) a_{W}\left(C, T_{F}^{*}\right)$

which can be rewritten as

$\ln \left(p_{W}\left(C, T_{F}^{*}\right)\right)=\ln \left(p_{W}\left(0, T_{F}\right)\right)+\ln \left(a_{W}\left(C, T_{F}^{*}\right)\right)$

The first and second terms on the right hand side of Eq. (A17) can be readily calculated using Eqs. (A2) and (A5), respectively. Fig. A3

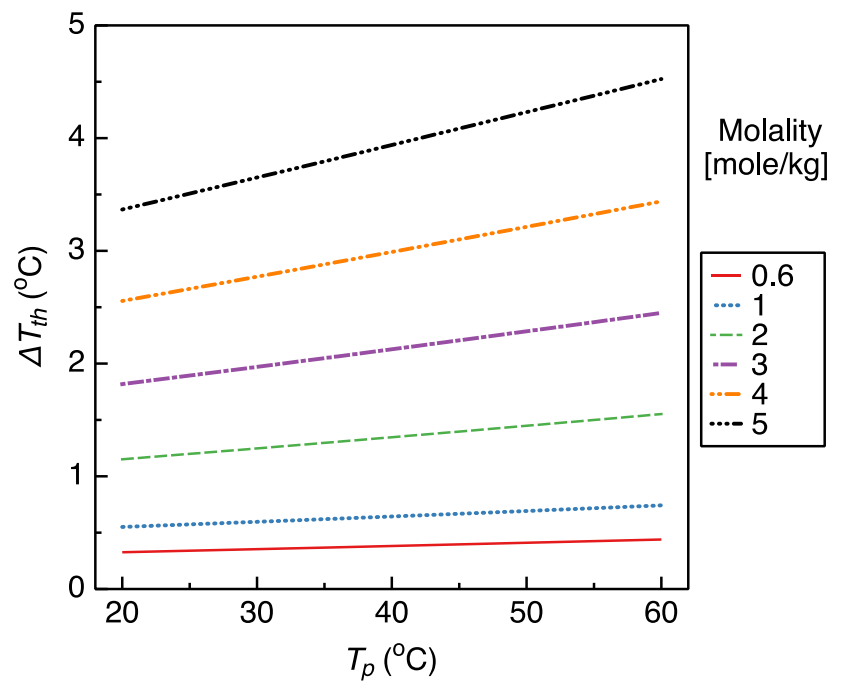

Fig. A3. Threshold temperature as a function of $\mathrm{NaCl}$ concentration and permeate temperature $T_{P}$. The curve in red is used in this paper. (For interpretation of the references to color in this figure legend, the reader is referred to the web version of this article.)
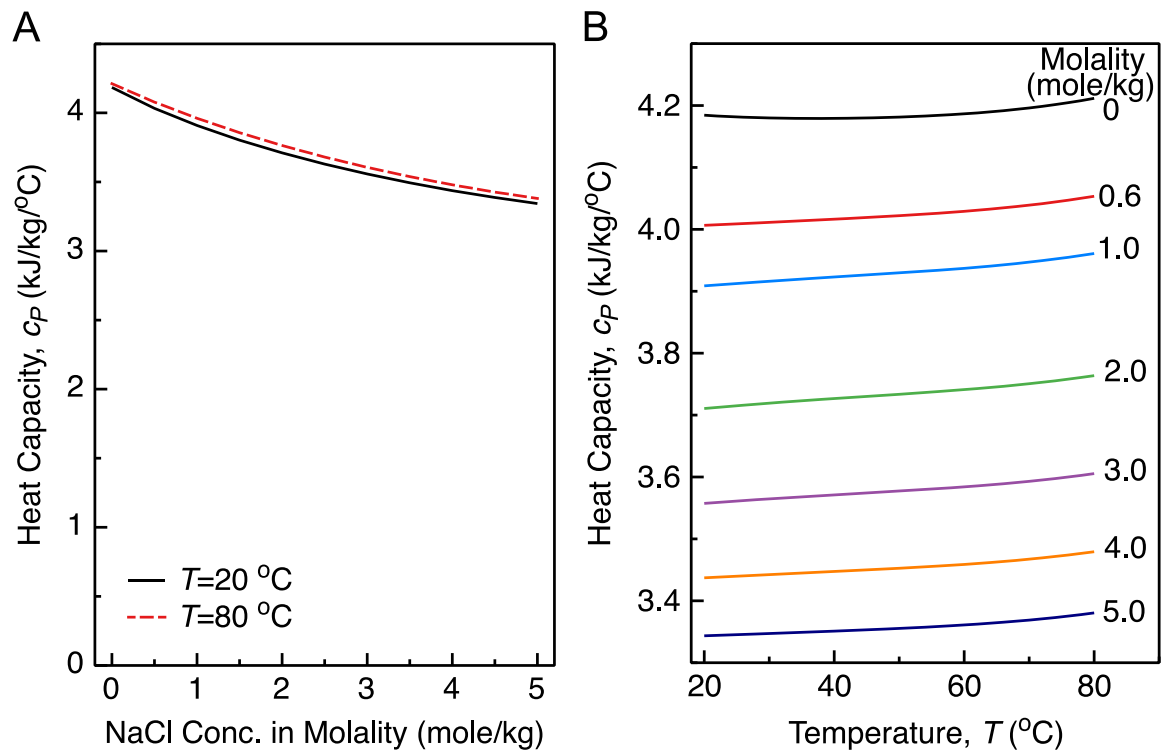

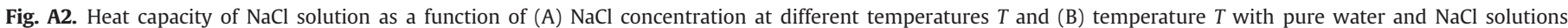
of different concentrations in molality. 
shows $\Delta T_{\text {th }}$ as a function of $C$ and $T_{P}$ based on Eq. (A17). For a given concentration, $\Delta T_{t h}$ is numerically linearly correlated to $T_{P}$ :

$\Delta T_{\text {th }}=m T_{P}+n$

The coefficients $m$ and $n$ in Eq. (A18) can be correlated to the concentration $C$ using the following equations $\left(R^{2}=1\right.$ for both correlations):

$m=2.689 \times 10^{-4} C^{2}+4.428 \times 10^{-3} C+8.847 \times 10^{-5}$

and

$n=3.024 \times 10^{-2} C^{2}+4.015 \times 10^{-1} C+2.032 \times 10^{-2}$

\section{Appendix C. Analytical analysis of the DCMD module}

Permeate limiting regime (PLR)

The overall heat balance of the permeate channel is given by

$c_{p, P}\left(Q_{P}^{f} T_{P}^{f}-Q_{P}^{0} T_{P}^{0}\right) \approx\left(\bar{h}_{v a p, P}+c_{p, P} \bar{T}_{P}\right) \Delta Q_{M D}$

The left hand side represents the change of heat flow in the permeate channel, whereas the right hand side represent the approximate heat transfer across the membrane along the entire module, with $\bar{h}_{v a p, P}$ being the averaged specific enthalpy of vaporization of the permeate stream and can be approximated as

$\bar{h}_{\text {vap }, P} \approx \frac{h_{\text {vap }}\left(0, T_{C}\right)+h_{\text {vap }}\left(0, T_{H}^{*}\right)}{2}$

$\bar{T}_{P}$ is the average temperature in the permeate channel and can be approximated as

$\bar{T}_{P} \approx \frac{T_{C}+T_{H}^{*}}{2}$

The reason of using $T_{C}$ and $T_{H}^{*}$ in Eqs. (A22) and (A23) is based on the fact that in the PLR the permeate temperature distribution covers the whole range of temperatures, from $T_{C}$ to $T_{H}^{*}$. Eqs. (A22) and (A23) are only approximations primarily because the transmembrane mass flux is not evenly distributed with $T_{P}$. However, these approximations seem to be reasonable as reflected by the remarkable consistence between the analytical results and the numerical results presented in Fig. 3 of the main text. The conductive heat transfer is intentionally neglected as (i) it is very small compared to the convective heat transfer, and (ii) it is dependent on the membrane area and thus its relation with $\Delta Q_{M D}$ cannot be defined.

Based on the definition of $\alpha$ and $\gamma$, Eq. (A21) can be rewritten as

$c_{p, P}\left((\alpha+\gamma) T_{P}^{f}-\alpha T_{P}^{0}\right) \approx\left(\bar{h}_{v a p, P}+c_{p, P} \bar{T}_{P}\right) \gamma$

Rearranging Eq. (A24) leads to

$\gamma \approx \frac{\alpha c_{p, P}\left(T_{P}^{f}-T_{P}^{0}\right)}{\bar{h}_{\text {vap }, P}+c_{p, P} \bar{T}_{P}-c_{p, P} T_{P}^{f}}$

Simplifying Eq. (A25) and expressing it as a function of the working temperature results in an expression that predicts the mass recovery rate of the feed:

$\gamma_{P L R} \approx \alpha \frac{T_{H}^{*}-T_{C}}{\left(\bar{h}_{\text {vap }, P} / c_{p, P}\right)-\left(\left(T_{H}^{*}-T_{C}\right) / 2\right)}$

To quantify the condition with which the operation is in PLR, we need to realize that in PLR the effluent temperature of the feed is higher than $T_{C}^{*}$. The overall heat balance of the feed channel is given by

$c_{p, F}\left(Q_{F}^{0} T_{F}^{0}-Q_{F}^{f} T_{F}^{f}\right) \approx\left(\bar{h}_{v a p, P}+c_{p, P} \bar{T}_{P}\right) \Delta Q_{M D}$

The left hand side represents the change of heat flow in the feed channel, where the right hand side approximately accounts for the heat flow across the membrane in the whole module. Note that we use the property of the permeate stream for the trans-membrane heat flow because in the PLR only the permeate temperature spans the entire temperature range from $T_{C}$ to $T_{H}^{*}$, whereas the effluent temperature of the feed is unknown without running a numerical simulation. Although the heat flow leaving the feed is theoretically not exactly the same as that arriving at the permeate, the difference is numerically negligible as have been discussed in the main text.

Eq. (A27) can be rewritten by using $\gamma$ to eliminate the flow rates:

$c_{p, F}\left[T_{F}^{0}-(1-\gamma) T_{F}^{f}\right] \approx\left(\bar{h}_{v a p, P}+c_{p, P} \bar{T}_{P}\right) \gamma$

We can then write an analytical expression of $T_{F}^{f}$ based on Eq. (A25):

$T_{F}^{f} \approx \frac{c_{p, F} T_{F}^{0}-\left(\bar{h}_{v a p, P}+c_{p, P} \bar{T}_{P}\right) \gamma}{c_{p, F}(1-\gamma)}$

Combining Eqs. (A26) and (A29) and using the condition for PLR ( $T_{F}^{f} \geq T_{C}^{*}$ ) leads to

$\alpha \leq \alpha_{P L R}=\frac{T_{H}-T_{C}^{*}}{T_{H}^{*}-T_{C}} \frac{\left(\bar{h}_{v a p, p} / c_{p, P}\right)-\left(\left(T_{H}^{*}-T_{C}\right) / 2\right)}{\left(\bar{h}_{\text {vap }, p} / c_{p, F}\right)+\left(c_{p, P} / c_{p, F}\right)\left(\left(T_{H}+T_{c}^{*}\right) / 2\right)-T_{C}^{*}}$

where $\alpha_{P L R}$ is the critical relative flow rate below which the operation is in PLR. Note that all variables on the right hand side are properties of the solution and/or the working temperatures. Therefore, for any given system, the $\alpha_{P L R}$ can be analytically determined.

Feed limiting regime (FLR)

The derivation of the analytical expressions for $\gamma_{F L R}$ and $\alpha_{F L R}$ is analogous to that for $\gamma_{P L R}$ and $\alpha_{P L R}$. Therefore we do not repeat the textual explanation in details, but simply present the key equations used in the derivation.

The overall heat balance of the feed can be written as

$c_{p, F}\left(Q_{F}^{0} T_{F}^{0}-Q_{F}^{f} T_{F}^{f}\right) \approx\left(\bar{h}_{v a p, F}+c_{p, P} \bar{T}_{F}\right) \Delta Q_{M D}$

where $\bar{h}_{v a p, F}$ and $\bar{T}_{F}$ are defined as

$\bar{h}_{\text {vap }, F} \approx \frac{h_{v a p}\left(C, T_{C}^{*}\right)+h_{\text {vap }}\left(C, T_{H}\right)}{2}$

and

$\bar{T}_{F} \approx \frac{T_{C}^{*}+T_{H}}{2}$

Eliminating the flow rates in Eq. (A31) using $\alpha$ and $\gamma$ yields

$c_{p, F}\left[T_{F}^{0}-(1-\gamma) T_{F}^{f}\right] \approx\left(\bar{h}_{v a p, F}+c_{p, P} \bar{T}_{F}\right) \gamma$

Note that the heat capacity on the right hand side is $c_{p, P}$ instead of $c_{p, F}$. Rearranging Eq. (A34) and replacing $T_{F}^{f}$ with $T_{C}^{*}, T_{F}^{0}$ with $T_{H}$ and $\bar{T}_{F}$ with $\left(T_{C}^{*}+T_{H}\right) / 2$ leads to the analytical expression for the mass recovery rate in the FLR:

$\gamma_{F L R} \approx \frac{T_{H}-T_{C}^{*}}{\left(\bar{h}_{v a p, F} / c_{p, F}\right)+\left(c_{p, P} / c_{p, F}\right)\left(\left(T_{H}+T_{C}^{*}\right) / 2\right)-T_{C}^{*}}$

The overall heat balance in the permeate channel can be expressed as

$c_{p, P}\left(Q_{P}^{f} T_{P}^{f}-Q_{P}^{0} T_{P}^{0}\right) \approx\left(\bar{h}_{v a p, F}+c_{p, P} \bar{T}_{F}\right) \Delta Q_{M D}$

Eliminating the flow rates in Eq. (A36) using $\alpha$ and $\gamma$ yields

$c_{p, P}\left[(\alpha+\gamma) T_{P}^{f}-\alpha T_{P}^{0}\right] \approx\left(\bar{h}_{v a p, F}+c_{p, P} \bar{T}_{F}\right) \gamma$

The condition for FLR is $T_{P}^{f} \leq T_{H}^{*}$, which after combining with 


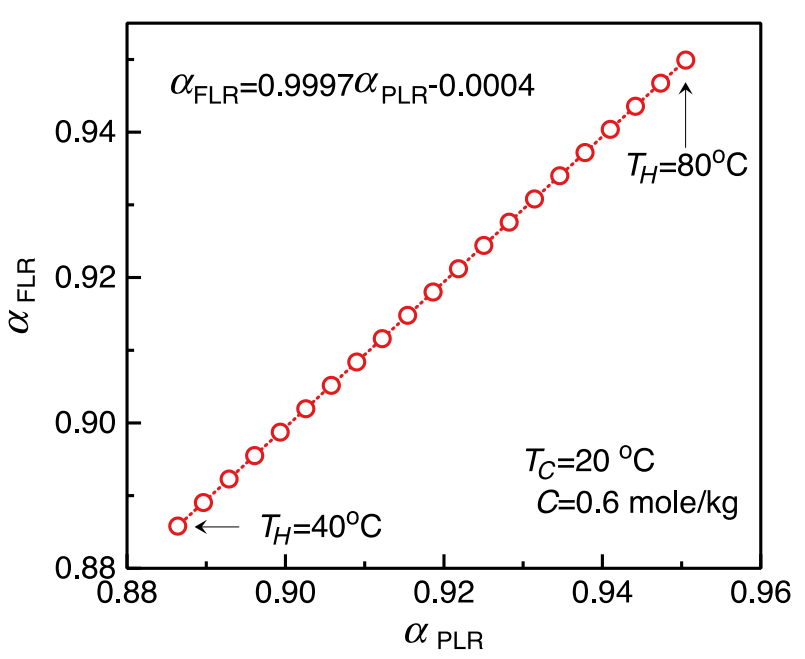

Fig. A4. Comparison between $\alpha_{P L R}$, as determined by Eq. (A30), and $\alpha_{F L R}$, as determined by Eq. (A38). A slope very close to 1.0 signifies the equivalence between $\alpha_{P L R}$ and $\alpha_{F L R}$. Here only the temperature of the heat source is varied. However, it can be easily shown that such an equivalence applies in other conditions.

Eqs. (A35) and (A37), leads to

$\alpha \geq \alpha_{F L R}=\frac{T_{H}-T_{C}^{*}}{T_{H}^{*}-T_{C}} \frac{\left(\bar{h}_{v a p, F} / c_{p, P}\right)+\left(\left(T_{H}+T_{C}^{*}\right) / 2\right)-T_{H}^{*}}{\left(\bar{h}_{v a p, F} / c_{p, F}\right)+\left(c_{p, P} / c_{p, F}\right)\left(\left(T_{H}+T_{c}^{*}\right) / 2\right)-T_{C}^{*}}$

Equivalence between $\alpha_{P L R}$ and $\alpha_{F F L R}$

Due to the approximations used in the derivations, Eqs. (A30) and (A38) are of different forms mathematically. However, in theory there should be a single critical $\alpha$ that divides the PLR and the FLR for unlimited mass transfer (i.e. large $\chi$ ). This can be confirmed by numerically comparing the $\alpha_{P L R}$ and $\alpha_{F L R}$ in different conditions, an example of which is given in Fig. A4.

\section{Appendix D. Temperature distribution profiles with the same mass transfer kinetics parameter $\chi$}

See Fig. A5 and Table A3.

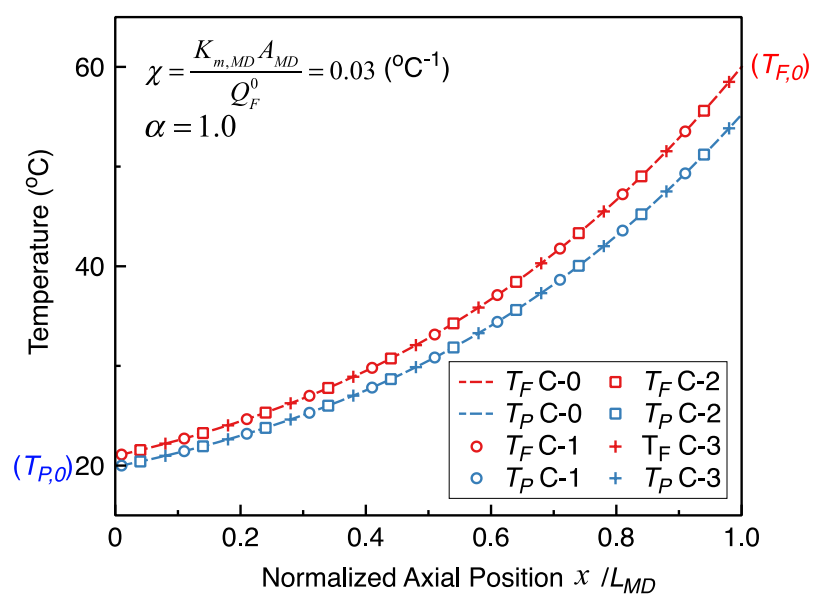

Fig. A5. Temperature distributions for feed and permeate flow in the MD module with different combinations of parameters that lead to a $\chi=0.03$. The details of parameter combinations can be found in Table A3. It shows that as long as $\chi$ is kept constant, the temperature distributions in the MD module will be the same.
Table A3

Details of parameter combinations used in the calculations for Fig. A5.

\begin{tabular}{llll}
\hline & $\boldsymbol{K}_{\boldsymbol{m}, \boldsymbol{M D}}\left(\mathrm{kg} \mathrm{m}^{2} \mathrm{~K}^{-1} \mathrm{~min}^{-1}\right)$ & $\boldsymbol{A}_{\boldsymbol{M D}}(\mathrm{m})$ & $\boldsymbol{Q}_{\boldsymbol{F}, \mathbf{0}}\left(\mathrm{kg} \mathrm{min}^{-1}\right)$ \\
\hline C-0 (reference) & 0.03 & 50 & 50 \\
$\mathbf{C - 1}$ & 0.06 & - & 100 \\
$\mathbf{C - 2}$ & 0.06 & 25 & - \\
$\mathbf{C - 3}$ & - & 100 & 100 \\
\hline
\end{tabular}

- $\alpha=1.0, T_{H}=60{ }^{\circ} \mathrm{C}, T_{C}=20^{\circ} \mathrm{C}$

- The "-" means the same values as in C-0 (reference combination)

- All combinations satisfy the requirement that $\chi=0.03$

\section{Nomenclature}

$A_{M D} \quad$ membrane area in the MD module $\left(\mathrm{m}^{2}\right)$

$A_{H X} \quad$ exchange area in the HX module $\left(\mathrm{m}^{2}\right)$

C electrolyte concentration (in molality) of the $\mathrm{NaCl}$ solution $\left(\mathrm{mol} \mathrm{kg}^{-1}\right)$

$C_{p, L}(C)$ heat capacity of $\mathrm{NaCl}$ solution of concentration $C$ $\left(\mathrm{kJ} \mathrm{kg}^{-1}{ }^{\circ} \mathrm{C}^{-1}\right)$

$c_{p, V} \quad$ heat capacity of water vapor $\left(\mathrm{kJ} \mathrm{kg}^{-1}{ }^{\circ} \mathrm{C}^{-1}\right)$

$c_{p, P}, c_{p, F}$ heat capacity of the permeate and feed in MD, respectively $\left(\mathrm{kJ} \mathrm{kg}^{-1}{ }^{\circ} \mathrm{C}^{-1}\right)$

$c_{p, h}, c_{p, c}$ heat capacity of the hot stream and cold stream in $\mathrm{HX}$, respectively $\left(\mathrm{kJ} \mathrm{kg}^{-1}{ }^{\circ} \mathrm{C}^{-1}\right)$

$h_{L, W}(T) \quad$ specific enthalpy of liquid water at temperature $T$ $\left(\mathrm{kJ} \mathrm{kg}^{-1}\right)$

$h_{L}(C, T) \quad$ specific enthalpy of an $\mathrm{NaCl}$ solution (of concentration $C)$ at temperature $T\left(\mathrm{~kJ} \mathrm{~kg}^{-1}\right)$

$h_{V}(T) \quad$ specific enthalpy of water vapor at temperature $T$ $\left(\mathrm{kJ} \mathrm{kg}^{-1}\right)$

$h_{\text {vap }}(C, T)$ specific enthalpy of vaporization of an $\mathrm{NaCl}$ solution (of concentration $C$ ) at temperature $T\left(\mathrm{~kJ} \mathrm{~kg}^{-1}\right)$

$\bar{h}_{v a p, F} \quad$ average specific enthalpy of vaporization of the feed stream, defined by Eq. (38) $\left(\mathrm{kJ} \mathrm{kg}^{-1}\right)$

$\bar{h}_{\text {vap,P }} \quad$ average specific enthalpy of vaporization of the permeate stream, defined by Eq. (35) $\left(\mathrm{kJ} \mathrm{kg}^{-1}\right)$

$h_{v a p, W}(T)$ specific enthalpy of vaporization of water at temperature $T\left(\mathrm{~kJ} \mathrm{~kg}^{-1}\right)$

$J_{m} \quad$ trans-membrane mass flux $\left(\mathrm{kg} \mathrm{m}^{-2} \mathrm{~min}^{-1}\right)$

$k_{c, M D} \quad$ thermal conductivity of the MD membrane matrix $\left(\mathrm{kJ} \mathrm{m}{ }^{-1} \min ^{-1}{ }^{\circ} \mathrm{C}^{-1}\right.$ )

$K_{m, M D} \quad$ mass transfer coefficient in $\mathrm{MD}\left(\mathrm{kg} \mathrm{m}^{-2} \min ^{-1}{ }^{\circ} \mathrm{C}^{-1}\right)$

$K_{c, M D}$ conductive heat trasnfer coefficient in MD $\left(\mathrm{W} \mathrm{m} \mathrm{m}^{-2} \mathrm{C}^{-1}\right.$ )

$K_{c, H X}$ heat transfer coefficient in $\mathrm{HX}\left(\mathrm{W} \mathrm{m}^{-2}{ }^{\circ} \mathrm{C}^{-1}\right)$

$L_{M D} \quad$ length of the MD module (m)

$L_{H X} \quad$ length of the HX module (m)

$p_{W}(C, T)$ partial vapor pressure of an $\mathrm{NaCl}$ solution (of concentration $C$ ) at temperature $T(\mathrm{kPa})$

$q_{C} \quad$ heat transferred to the heat sink to bring the stream temperature to $T_{C}(\mathrm{~kJ})$

$q_{H} \quad$ heat supplied by the heat source to bring the stream temperature to $T_{H}(\mathrm{~kJ})$

$q_{m} \quad$ trans-membrane heat flux in $\mathrm{MD}\left(\mathrm{kJ} \mathrm{m}^{-2} \mathrm{~min}^{-1}\right)$

$Q_{i} \quad$ mass flow rate of stream $i\left(\mathrm{~kg} \mathrm{~min}^{-1}\right)$

$Q_{H}, Q_{C} \quad$ mass flow rates of the hot stream and the cold stream in the HX module, respectively $\left(\mathrm{kg} \mathrm{min}^{-1}\right)$

$Q_{F}, Q_{P} \quad$ mass flow rates of the feed and the permeate streams in the MD module, respectively $\left(\mathrm{kg} \mathrm{min}^{-1}\right)$

$S_{i} \quad$ stream $i$ in the MD-HX system diagram (Fig. 1A)

$T_{i} \quad$ temperature of stream $i\left({ }^{\circ} \mathrm{C}\right)$ 
$T_{F}, T_{P} \quad$ temperatures of the feed and permeate streams in $\mathrm{MD}$, respectively $\left({ }^{\circ} \mathrm{C}\right)$

$T_{F}^{0}, T_{P}^{0} \quad$ influent temperatures of the feed and permeate streams in MD, respectively (Fig. $1 \mathrm{C}$ ) $\left({ }^{\circ} \mathrm{C}\right)$

$T_{F}^{f}, T_{P}^{f} \quad$ effluent (final) temperatures of the feed and permeate streams in MD, respectively (Fig. 1C) $\left({ }^{\circ} \mathrm{C}\right.$ )

$T_{h}, T_{c}$ temperatures of the hot and the cold stream in HX, respectively $\left({ }^{\circ} \mathrm{C}\right)$

$T_{h}^{0}, T_{c}^{0} \quad$ influent temperatures of the hot and the cold streams in $\mathrm{HX}$, respectively $\left({ }^{\circ} \mathrm{C}\right)$

$T_{h}^{f}, T_{c}^{f} \quad$ effluent temperatures of the hot and the cold streams in $\mathrm{HX}$, respectively $\left({ }^{\circ} \mathrm{C}\right)$

$T_{H}, T_{C}$ temperatures of the heat source and heat sink, respectively $\left({ }^{\circ} \mathrm{C}\right)$

$T_{H}^{*}(C) \quad$ temperature of pure water at which its partial vapor pressure is equal to the water partial vapor pressure of an $\mathrm{NaCl}$ solution (of concentration $C$ ) at temperature $T_{H}\left({ }^{\circ} \mathrm{C}\right)$

$T_{C}^{*}(C)$ temperature of an $\mathrm{NaCl}$ solution (of concentration C) at which its water partial vapor pressure is equal to the partial vapor pressure of pure water at temperature $T_{C}\left({ }^{\circ} \mathrm{C}\right)$

$T_{F}^{*}\left(C, T_{P}\right)$ temperature of the feed in MD (of an $\mathrm{NaCl}$ concentration $C$ ) at which the partial vapor pressure is equal to that of the permeate at $T_{P}\left({ }^{\circ} \mathrm{C}\right)$

$T_{P}^{*}\left(C, T_{F}\right)$ temperature of the permeate in MD at which the partial vapor pressure is equal to that of the feed (of an $\mathrm{NaCl}$ concentration $\mathrm{C}$ ) at $T_{P}^{*}\left({ }^{\circ} \mathrm{C}\right)$

$\Delta T$ difference between the feed and permeate temperatures $\left({ }^{\circ} \mathrm{C}\right)$

$\Delta T_{t h} \quad$ threshold temperature difference at which the partial vapor pressures of the feed and the permeate in MD are the same $\left({ }^{\circ} \mathrm{C}\right)$

$x \quad$ axial position along the MD or HX module ( $\mathrm{m}$ )

$z \quad$ position in the direction normal to the membrane in $\mathrm{MD}(\mathrm{m})$

\section{Greek symbols}

$\alpha \quad$ relative mass flow rate between the permeate and the feed in MD, defined by Eq. (31) (dimensionless) relative mass flow rate above which the MD operation is in feed limiting regime, defined by Eq. (37) (dimensionless)

$\alpha_{P L R} \quad$ relative mass flow rate below which the MD operation is in permeate limiting regime, defined by Eq. (34) (dimensionless)

$\alpha^{*} \quad$ the critical relative flow rate dividing the FLR and PLR in $\mathrm{MD},\left(\alpha^{*} \approx \alpha_{F L R} \approx \alpha_{P L R}\right)$ (dimensionless)

$\beta \quad$ specific heat duty-the energy input required to produce a unit mass of distillate, defined by Eq. (26) $\left(\mathrm{kJ} \mathrm{kg}^{-1}\right)$

$\chi \quad$ mass transfer parameter in MD, defined by Eqs. (40) $\left({ }^{\circ} \mathrm{C}^{-1}\right)$

thickness of the MD membrane (m)

porosity of the MD membrane (dimensionless)

mass recovery rate of the feed, defined by Eq. (25)

(dimensionless)

heat recovery efficiency of the MD-HX system (dimensionless)

relative importance of conductive heat transfer as compared to the convective heat transfer, defined by Eq. (43) dimensionless

$\zeta_{F} \quad$ conductive heat flux at the feed-membrane interface $\left(\mathrm{kJ} \mathrm{m}^{-2} \mathrm{~min}^{-1}\right)$
$\zeta_{P}$

conductive heat flux at the permeate-membrane interface $\left(\mathrm{kJ} \mathrm{m}^{-2} \mathrm{~min}^{-1}\right)$

$\Delta \zeta \quad$ the difference between $\zeta_{P}$ and $\zeta_{F}\left(\mathrm{~kJ} \mathrm{~m}^{-2} \mathrm{~min}^{-1}\right)$

\section{References}

[1] M.S. Khayet, T. Matsuura, Membrane distillation: principles and applications, 2011.

[2] A.M. Alklaibi, N. Lior, Membrane-distillation desalination: status and potential, Desalination 171 (2005) 111-131.

[3] Q. Ge, P. Wang, C. Wan, T.-S. Chung, Polyelectrolyte-promoted forward osmosis-membrane distillation (FO-MD) hybrid process for dye wastewater treatment, Environ. Sci. Technol. 46 (2012) 6236-6243.

[4] M. Xie, L.D. Nghiem, W.E. Price, M. Elimelech, A Forward, Osmosis-membrane distillation hybrid process for direct sewer mining: system performance and limitations, Environ. Sci. Technol., http://dx.doi.org/10.1021/es404056e, in press.

[5] B.E. Logan, M. Elimelech, Membrane-based processes for sustainable power generation using water, Nature 488 (2012) 313-319(Nature Publishing Group).

[6] K.Y. Wang, M.M. Teoh, A. Nugroho, T.-S. Chung, Integrated forward osmosismembrane distillation (FO-MD) hybrid system for the concentration of protein solutions, Chem. Eng. Sci. 66 (2011) 2421-2430.

[7] K.W. Lawson, D.R. Lloyd, Membrane distillation, J. Membr. Sci. (1997) 1-25.

[8] R.B. Saffarini, E.K. Summers, H.A. Arafat, J.H. Lienhard V., Economic evaluation of stand-alone solar powered membrane distillation systems, Desalination 299 (2012) 55-62.

[9] N. Ghaffour, T.M. Missimer, G.L. Amy, Technical review and evaluation of the economics of water desalination: current and future challenges for better water supply sustainability, Desalination 309 (2013) 197-207.

[10] A. Alkhudhiri, N. Darwish, N. Hilal, Membrane distillation: a comprehensive review, Desalination 287 (2012) 2-18.

[11] K.W. Lawson, D.R. Lloyd, Membrane distillation. II. Direct contact MD, J. Membr. Sci. 120 (1996) 123-133.

[12] E. Curcio, E. Drioli, Membrane distillation and related operations-a review Sep. Purif. Rev. 34 (2005) 35-86.

[13] E. Guillén-Burrieza, J. Blanco, G. Zaragoza, D.-C. Alarcón, P. Palenzuela, M. Ibarra, et al., Experimental analysis of an air gap membrane distillation solar desalination pilot system, J. Membr. Sci. 379 (2011) 386-396.

[14] M. Khayet, P. Godino, J.I. Mengual, Theory and experiments on sweeping gas membrane distillation, J. Membr. Sci. 165 (2000) 261-272.

[15] S. Bandini, A. Saavedra, G.C. Sarti, Vacuum membrane distillation: experiments and modeling, AIChE J. 43 (1997) 398-408.

[16] M.S. El-Bourawi, Z. Ding, R. Ma, M. Khayet, A framework for better understanding membrane distillation separation process, J. Membr. Sci. 285 (2006) $4-29$.

[17] R.W. Schofield, A.G. Fane, C. Fell, Heat and mass transfer in membrane distillation, J. Membr. Sci. 33 (1987) 299-313.

[18] M. Gryta, M. Tomaszewska, Heat transport in the membrane distillation process, J. Membr. Sci. 144 (1998) 211-222.

[19] J. Phattaranawik, R. Jiraratananon, Direct contact membrane distillation: effect of mass transfer on heat transfer, J. Membr. Sci. 188 (2001) 137-143.

[20] J. Phattaranawik, R. Jiraratananon, A.G. Fane, Heat transport and membrane distillation coefficients in direct contact membrane distillation, J. Membr. Sci. 212 (2003) 177-193.

[21] P. Termpiyakul, R. Jiraratananon, S. Srisurichan, Heat and mass transfer characteristics of a direct contact membrane distillation process for desalination, Desalination 177 (2005) 133-141.

[22] S. Srisurichan, R. Jiraratananon, A. FANE, Mass transfer mechanisms and transport resistances in direct contact membrane distillation process, J. Membr. Sci. 277 (2006) 186-194.

[23] A.M. Alklaibi, N. Lior, Heat and mass transfer resistance analysis of membrane distillation, J. Membr. Sci. 282 (2006) 362-369.

[24] M. Qtaishat, T. Matsuura, B. Kruczek, M. Khayet, Heat and mass transfer analysis in direct contact membrane distillation, Desalination 219 (2008) 272-292.

[25] L. Martınez-Díez, M.I. Vázquez-González, Study of membrane distillation using channel spacers, J. Membr. Sci. 144 (1998) 45-56.

[26] L. Martınez-Díez, M.I. Vázquez-González, A method to evaluate coefficients affecting flux in membrane distillation, J. Membr. Sci. 173 (2000) 225-234.

[27] J. Phattaranawik, R. Jiraratananon, A.G. Fane, C. Halim, Mass flux enhancement using spacer filled channels in direct contact membrane distillation, J. Membr. Sci. 187 (2001) 193-201.

[28] J. Phattaranawik, R. Jiraratananon, A.G. Fane, Effects of net-type spacers on heat and mass transfer in direct contact membrane distillation and comparison with ultrafiltration studies, J. Membr. Sci. 217 (2003) 193-206.

[29] T.Y. Cath, V.D. Adams, A.E. Childress, Experimental study of desalination using direct contact membrane distillation: a new approach to flux enhancement, J. Membr. Sci. 228 (2004) 5-16. 
[30] L. Song, B. Li, K.K. Sirkar, J.L. Gilron, Direct contact membrane distillationbased desalination: novel membranes, devices, larger-scale studies, and a model, Ind. Eng. Chem. Res. 46 (2007) 2307-2323.

[31] L.-H. Cheng, P.-C. Wu, J. Chen, Modeling and optimization of hollow fiber DCMD module for desalination, J. Membr. Sci. 318 (2008) 154-166.

[32] V.A. Bui, L.T.T. Vu, M.H. Nguyen, Modelling the simultaneous heat and mass transfer of direct contact membrane distillation in hollow fibre modules, J. Membr. Sci. 353 (2010) 85-93.

[33] G. Zuo, R. Wang, R. Field, A.G. Fane, Energy efficiency evaluation and economic analyses of direct contact membrane distillation system using Aspen Plus, Desalination 283 (2011) 237-244.

[34] E.K. Summers, H.A. Arafat, J.H. Lienhard, Energy efficiency comparison of single-stage membrane distillation (MD) desalination cycles in different configurations, Desalination 290 (2012) 54-66.

[35] A.G. Fane, R.W. Schofield, C. Fell, The efficient use of energy in membrane distillation, Desalination (1987) 231-243.

[36] R.W. Schofield, A.G. Fane, C.J.D. Fell, R. Macoun, Factors affecting flux in membrane distillation, Desalination 77 (1990) 279-294.

[37] J.C.S. Chou, A.M. Rowe Jr., Enthalpies of aqueous sodium chloride solutions from 32-350 ${ }^{\circ}$, Desalination 6 (1969) 105-115.

[38] I.S. Al-Mutaz, Potential of nuclear desalination in the Arabian Gulf countries, Desalination 135 (2001) 187-194.

[39] S.V. Patankar, Numerical heat transfer and fluid flow, 1980.

[40] A.R. Conn, N.I.M. Gould, P.L. Toint, Trust Region Methods, Society for Industrial and Applied Mathematics, Philadelphia, PA, USA, 2000.
[41] X. Ge, X. Wang, A simple two-parameter correlation model for aqueous electrolyte solutions across a wide range of temperatures, J. Chem. Eng. Data 54 (2009) 179-186.

[42] X. Ge, X. Wang, Estimation of freezing point depression, boiling point elevation, and vaporization enthalpies of electrolyte solutions, Ind. Eng. Chem. Res. 48 (2009) 2229-2235.

[43] M. Laliberté, A model for calculating the heat capacity of aqueous solutions, with updated density and viscosity data, J. Chem. Eng. Data 54 (2009) 1725-1760.

[44] F. Kreith, R.M. Manglik, M.S. Bohn, Principles of Heat Transfer, Cengage Learning, Florence, KY, USA, 2010.

[45] T.L. Bergman, A.S. Lavine, F.P. Incropera, D.P. DeWitt, Fundamental of Heat and Mass Transfer, 7th Edition, Wiley, USA, 2011

[46] L. Song, J.Y. Hu, S.L. Ong, W.J. Ng, M. Elimelech, M. Wilf, Emergence of thermodynamic restriction and its implications for full-scale reverse osmosis processes, Desalination 155 (2003) 213-228.

[47] L. Song, J.Y. Hu, S.L. Ong, W.J. Ng, M. Elimelech, M. Wilf, Performance limitation of the full-scale reverse osmosis process, J. Membr. Sci. 214 (2003) 239-244.

[48] H. Lee, F. He, L. Song, J. Gilron, K.K. Sirkar, Desalination with a cascade of crossflow hollow fiber membrane distillation devices integrated with a heat exchanger, AIChE J. (2011).

[49] M. Elimelech, W.A. Phillip, The future of seawater desalination: energy, technology, and the environment, Science 333 (2011) 712-717. 